\title{
Extracellular Enzymes and Bioactive Compounds from Antarctic Terrestrial Fungi for Bioprospecting
}

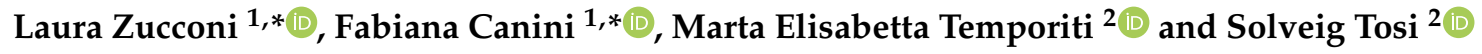 \\ 1 Department of Ecological and Biological Sciences, University of Tuscia, Largo dell'Università snc, \\ 01100 Viterbo, Italy \\ 2 Department of Earth and Environmental Sciences, University of Pavia, via S. Epifanio 14, 27100 Pavia, Italy; \\ martaelisabett.temporiti01@universitadipavia.it (M.E.T.); solveig.tosi@unipv.it (S.T.) \\ * Correspondence: zucconi@unitus.it (L.Z.); canini.fabiana@unitus.it (F.C.); \\ Tel.: +39-328-2741247 (L.Z.); +39-347-9288247 (F.C.)
}

Received: 21 July 2020; Accepted: 2 September 2020; Published: 4 September 2020

check for updates

\begin{abstract}
Antarctica, one of the harshest environments in the world, has been successfully colonized by extremophilic, psychrophilic, and psychrotolerant microorganisms, facing a range of extreme conditions. Fungi are the most diverse taxon in the Antarctic ecosystems, including soils. Genetic adaptation to this environment results in the synthesis of a range of metabolites, with different functional roles in relation to the biotic and abiotic environmental factors, some of which with new biological properties of potential biotechnological interest. An overview on the production of cold-adapted enzymes and other bioactive secondary metabolites from filamentous fungi and yeasts isolated from Antarctic soils is here provided and considerations on their ecological significance are reported. A great number of researches have been carried out to date, based on cultural approaches. More recently, metagenomics approaches are expected to increase our knowledge on metabolic potential of these organisms, leading to the characterization of unculturable taxa. The search on fungi in Antarctica deserves to be improved, since it may represent a useful strategy for finding new metabolic pathways and, consequently, new bioactive compounds.
\end{abstract}

Keywords: adaptative strategies; bioprospecting; extreme environment; soil fungi

\section{Introduction}

Antarctica is characterized by the coldest and driest climate known on Earth. Within this environment, the Antarctic Peninsula and the adjacent islands (maritime Antarctica) have a cold maritime climate, with mean monthly temperatures from 0 to less than $-15^{\circ} \mathrm{C}$, and annual precipitations from 350 to over $500 \mathrm{~mm}$. More extreme conditions characterize the rest of the continent (continental Antarctica), with milder conditions along the coasts, becoming more extreme moving towards inland sites, up to mean monthly temperatures from -15 to $-50{ }^{\circ} \mathrm{C}$ and nearly non-existent precipitation on the Ice Plateau [1]. These differences result in a greater biodiversity characterizing the maritime Antarctica and the coastal sites of continental Antarctica than the inner locations. However, in all these Antarctic regions the terrestrial ecosystems are dominated, more than those of other continents, by microorganisms, characterized by astonishing adaptations to withstand the extreme conditions of the environment [2,3]. In particular, they have to cope with extremely low temperatures, wide temperature fluctuations, frequent freeze and thaw events during the austral summer, strong winds, intense solar and UV irradiations, different $\mathrm{pH}$ conditions, desiccation, and locally high salinity. Microorganisms spread on the limited different substrates available, such as soils, rocks, bird feathers and dung, bryophytes, and lichens, that are sheltered niches, characterized by more buffered conditions [3-5]. 
Among microbes inhabiting soil ecosystems, fungi are ubiquitous and mainly responsible for the breaking down of organic matter and the releasing of phosphorus, nitrogen and carbon into the atmosphere and soil. They also produce and secrete a wide variety of enzymes and bioactive secondary metabolites, some of which of great biotechnological interest. Some fungal species found in the Antarctic environment are possibly present as air transported propagules from outside the continent and do not actively grow in this natural environment. Other species are indigenous, or even endemic, able to actively grow and reproduce in environmental conditions that are accounted among the most extreme on Earth $[4,6,7]$. These latter organisms have mostly attracted the interest of the scientific community as, to survive to this combination of environmental constraints, had to have developed multiple mechanisms of stress tolerance. These mechanisms include the activation of peculiar metabolic pathways and the production of either enzymes active at temperatures below the common limits, or other bioactive compounds of great potential value for biotechnological applications [7]. In this optic, Antarctica represents a very attractive location to search for novel cold-adapted enzymes or bioactive compounds both for being a permanently cold environment and because of the minimum human-associated activity.

Recent molecular and phylogenetic studies carried out on Antarctic and Arctic soils, sea ice cores, and cyanobacterial mats suggest that microbial diversity in harsh cold habitats may be much greater than ever appreciated [8,9]. However, even if culture-independent molecular methods, such as DNA metabarcoding, have revolutionized our understanding of microbial ecology, making possible to define the actual microbial communities composition and functionality, the traditional cultural approaches make possible to obtain isolates to be tested and exploited for their metabolic capabilities. Therefore, given the biotechnological potential of microbial enzymes or other bioactive compounds, there is a need to increase efforts for greater species recovery, as suggested for bacteria [10]. Different bioactivities have been observed among isolates of the same species, as well as different secondary metabolites produced by conspecific isolates. Considering that, it is mandatory the preservation of isolated strains in culture collections, to have available wide and unique sources of new bioactive producers [11]. New interesting results may be gained using metagenomics approaches, both to identify functional traits that drive microbial survival and community assembly, and to identify genes involved in metabolic pathways of biotechnological interest. Anyway, as stated by Duarte et al. [12], metagenomics allows one to explore new environments for putative functions, but there is still a lack of information related to the prospecting of Antarctic enzymes based on the metagenomic approach. Among the few studies published to date, the review of de Pascale et al. [13] must be taken into account, where some enzymes such as esterase, lipase, $\beta$-galactosidase, $\alpha$-amilase and chitinase from polar bacteria were reported and discussed in the light of metagenomics screening. The metabolic competences of microbial communities inhabiting soil and rock surface niches in McKelvey Valley (McMurdo Dry Valleys) were also identified through GeoChip functional gene arrays and related to environmental stressors, offering tangible clues of the mechanisms behind the endurance of microorganisms in this inhospitable region [14]. Soils, in particular, comprised a unique reservoir of genes of particular applicative interest, such as those involved in transformation of organic hydrocarbons and in the lignin-like degradative pathways. Antibiotic resistance genes were also identified, indirectly indicating a level of competitive interactions between taxa, confirming data previously reported by shotgun metagenomics sequencing of these communities [15].

Despite the apparent simplicity or homogeneity of Antarctic terrestrial environments, microbial populations are often geographically structured into genetically distinct lineages. Fungi in pristine soils are more diverse than previously thought and their richness, abundance and composition are usually found to be mainly determined by environmental conditions, among which soil parameters seem to be the most prominent influencing factors $[5,16,17]$. In studies on Antarctic soils, the predominance of filamentous Ascomycota has been frequently reported, followed by Basidiomycota, mostly represented by yeasts; the other phyla (as Mucoromycota, Chytridiomycota, Mortierellomycota, and others) are less diverse and occasionally recorded [3-5,11,17]. 
The fungal component of continental Antarctic soils is mostly represented by few psychrophilic and many mesophilic-psychrotolerant and oligotrophic species. Among the physiological and morphological adaptations adopted by these microorganisms, the alteration of membrane lipid composition, the synthesis of different compatible solutes with protection roles of enzyme activity and with cryoprotective ability under complete dehydration (anhydrobiosis), the production of exopolysaccharides, and a strong melanin pigmentation are the most common [3].

Many studies have dealt with enzymes from cold-adapted Antarctic fungi and their potential applications, and many other research groups have reported inventory studies on species and strains able to produce bioactive prototype compounds. Given the large number of papers published, a probably not exhaustive overview is here provided; we restricted our interest to soil fungi and aimed to focus and exploit on the ecological significance of these compounds. A map of the collection sites is reported in Figure 1, from which it appears as Victoria Land in continental Antarctica and the Antarctic Peninsula and the South Shetland Islands in maritime Antarctica are the areas where the soil samples have been mostly collected, to search for enzymes and other compounds.

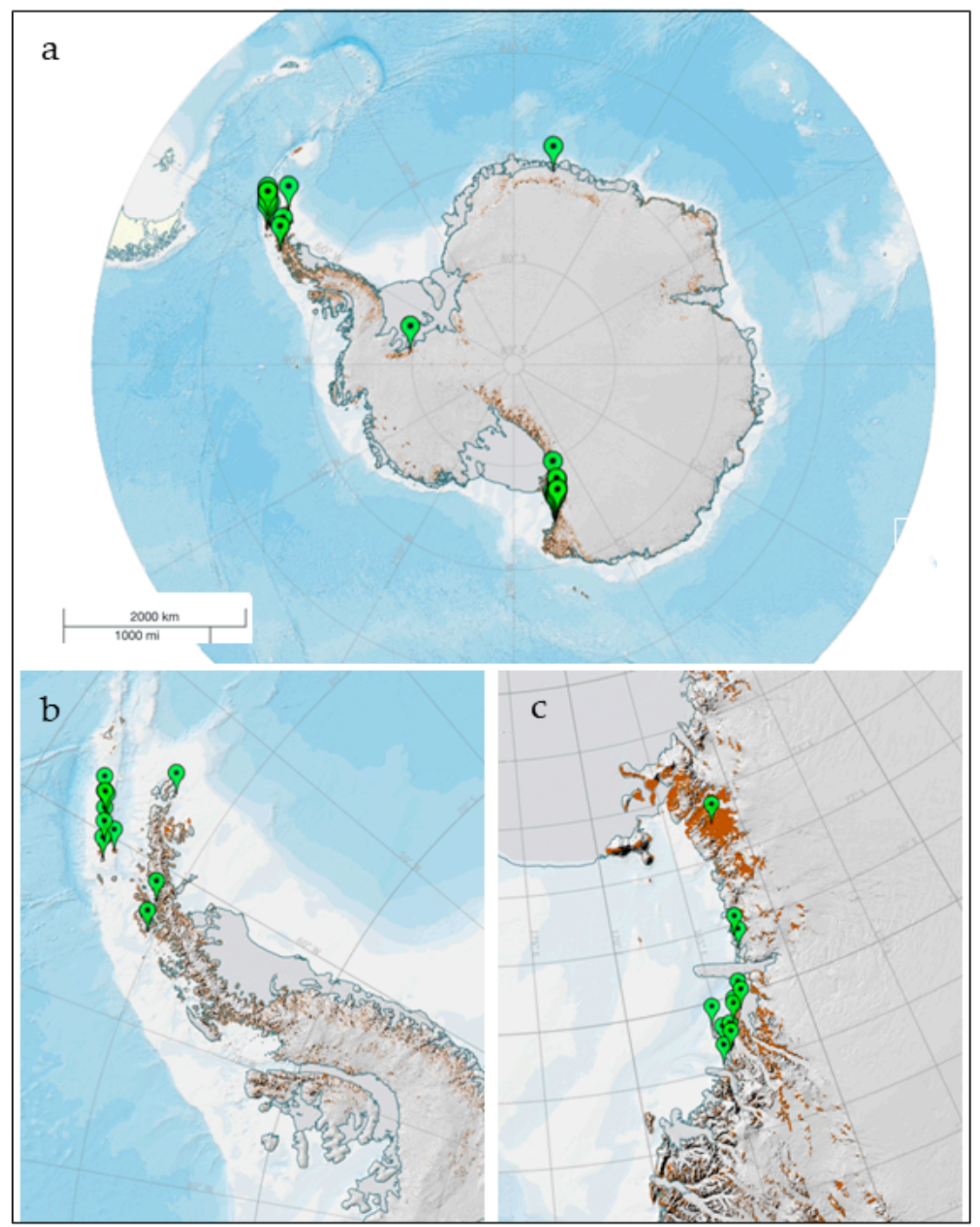

Figure 1. Map of the sites from where soil fungi have been isolated, producing enzymes and bioactive compounds (a); more detailed views of the most investigated areas, in maritime (b) and continental (c) Antarctica. Figure has been realized by the SCAR (Scientific Committee on Antarctic Research) Antarctic Digital Database (https://www.add.scar.org/). 


\section{Enzymes}

Cold-adapted enzymes produced by psychrophilic or psychrotolerant microorganisms are an important element for the survival strategy in Antarctic ecosystems. In fact, if extremely low temperatures generally restrict microbial enzyme activity, cold-active enzymes can conduct transformations at lower temperatures than those produced by their mesophilic homologues. They display a high specific activity at low and moderate temperatures, associated with a relatively high thermosensitivity [18]. These properties make them a potentially valuable alternative to their mesophilic counterparts in cold environments. They may represent an interesting advantage in large scale processes, that generally occur at higher temperatures, for reducing the energy costs associated with heating steps [19-21]. While the thermosensitivity provides the possibility of rapidly inactivating them by mild heat treatments, preserving in this way the product quality [18]. Some hypotheses have been proposed to explain the cold adaptation of these enzymes compared to their mesophilic and/or thermophilic counterparts, and amino acid substitutions was supposed as a possible mechanism. These properties with regard to their current and possible applications in biotechnology have been reviewed by Marx et al. [18]. Additionally, Antarctic enzymes often exhibit a wide range of $\mathrm{pH}$ and temperature optima [12]. This latter property is strictly linked to wide, frequent and sudden temperature variations experienced by terrestrial surfaces, based on soil expositions and weather conditions. These wide ranges force these microorganisms to adapt to different ecological niches. A list of the enzymes produced by Antarctic soil fungi are reported in Table 1 grouped for their biological activity. The strains for which the activities have been reported, with their isolation locality, and the temperature at which the enzymatic production has been tested are reported.

One of the widest screening for enzyme production by Antarctic fungi dates to 1997 when 33 fungal strains, belonging to 11 genera isolated from soil and mosses samples collected in different sites of Northern Victoria Land (continental Antarctica) were tested for their ability to produce a set of extracellular enzymes. The enzymes tested were among those related to the decomposition of common organic substrata: lipases, polygalacturonase, pectinlyase, amylase, cellulase, chitinase, phosphatase, glucose oxidase, urease, protease, and RNase [22]. Among these enzymes, lipases were generally produced in high quantities by almost all the strains; polygalacturonase, amylase and phosphatase were common, and glucose oxidase, protease and DNase were generally scarce or absent [22]. In particular, the psychrotolerant strain Lecanicillium muscarium (CCFEE 5003), formerly identified as Verticillium lecanii, resulted to be a powerful producer of cold-tolerant extracellular enzymes, among which chitin-hydrolyzing enzymes seemed to be the most abundant [22]. The chitinolytic activity was induced by the presence of chitin and other polysaccharides and was subjected to catabolite repression. The chitinolytic system of $L$. muscarium consisted of a number of different proteins with various molecular weights and different biochemical characteristics. Their most significant trait was the marked cold-tolerance and efficiency as mycoparasite against fungi and oomycetes. These properties make these enzymes useful to be exploited for the reuse of chitin-rich wastes and in biocontrol of pathogens [23]. These chitinolytic enzymes, probably also linked to the large amounts of chitin in ornithogenic soils due to the krill-rich diet of penguins [24], showed to be active over a broad range of temperatures [25], including very low temperatures $\left(5^{\circ} \mathrm{C}\right.$ or below) where other microorganism fail [23]. This ability to produce chitinases active at low temperatures could be very interesting in pharmaceutical industry such as for the preparation of single-cell protein, chitooligosaccharides, and N-acetyl D glucosamine. In addition, these enzymes are involved in the isolation of protoplasts from cells with chitinized walls such as fungi and yeasts, in the biological control of pathogenic fungi and insects, and in waste treatment (chitin is the second most abundant natural polymer on the Earth) [26]. A high cellulase activity at low temperature was also recorded in a Verticillium strain from King George Island soil. Its potential use for the development of economic and efficient systems for the bioconversion of lignocellulosic compounds into biofuels was stressed [27]. The production of ligninolytic enzymes was reported for the first time in Antarctica for fungi isolated by soil collected in different sites in the Admiralty Bay region (King George Island, Maritime Antarctica). Each characterized by different 
physicochemical compositions and fungal communities [28]. The NMDS (Non-metric multidimensional scaling) analysis showed fungal communities well separated by sampling site, each characterized by distinct soil physicochemical parameters. Besides differences in fungal communities composition, no correlations were computed between the enzymatic productions and the collection sites. In the face of a total of 891 isolates, the information on their enzymatic productions are very scant, with positive results mostly obtained by fungi isolated at $15{ }^{\circ} \mathrm{C}$ from root-associated soils of both Deschampsia antarctica and Colobanthus quitensis [28]. Fungi isolated from soils of an ornithogenic site close a penguin rookery, an undisturbed vegetated area, and a human impacted site at King George Island, were compared for their diversity, thermal characteristics (at 4 and $25^{\circ} \mathrm{C}$ ) and extracellular hydrolase enzyme production. Both cellulase and amylase activities at $25^{\circ} \mathrm{C}$ tended to be greater than that at $4{ }^{\circ} \mathrm{C}$, and both were stronger in strains isolated from the human impacted site, possibly related to an enrichment in soil organic matter. Interestingly, a greater number of strains isolated at 4 than at $25^{\circ} \mathrm{C}$ were significant cellulase producers [29].

Some Antarctic fungi are able to grow despite the very low organic matter content in the soil, thanks to their wide enzymatic pattern, that increases survival chances in unfavorable environmental conditions [22]. Enzymatic activities of dehydrogenase, $\beta$-glucosidase, acid and alkaline phosphatase and arylsulphatase were detected in soils of the Dry Valleys, both amended or not with additions of $\mathrm{C}$ and $\mathrm{N}$ in both simple and complex forms. The results indicated that mineralization of organic $\mathrm{C}, \mathrm{P}$ and $\mathrm{S}$ compounds toke place also in unamended soils, despite the very low organic matter content. The activities of almost all the enzymes were increased by $\mathrm{C}$ and combined $\mathrm{C}$ and $\mathrm{N}$ additions, and were either unchanged or reduced by the addition of either $\mathrm{N}$ only or $\mathrm{N}$ with only small $\mathrm{C}$ amounts. No evidence of shifts in the community structure as a result of the $\mathrm{C}$ and $\mathrm{N}$ supplementation was observed [30].

The high number of yeast strains isolated in Antarctica and the wide diversity of their enzyme activities highlighted the importance of these organisms in nutrient recycling in this environment. A number of yeast strains isolated from the rhizosphere of D. antarctica, ornithogenic (penguin guano) and not-ornithogenic soils, and other substrates in maritime Antarctica, were tested for their ability to produce secondary metabolites [31]. Cellulolytic and esterase were the most frequent activities, and most of the isolates $(60 \%)$ had extracellular enzymatic activities at 4 and $20^{\circ} \mathrm{C}$. Moreover, $41.7 \%$ of the yeast strains isolated were able to produce pigments and/or mycosporines. These observations confirmed again the Antarctic mycobiota as a rich source di metabolites, involved in their adaptation to the environment, with high potential applications. For example, esterase finds application in various fields, playing an important role in the pharmaceutical industries for synthesis of chiral drugs and being able to degrade both natural materials and industrial pollutants [32]. Cellulase and esterase are suitable for degrading both agricultural waste and toxic chemicals, making them an actual interesting tool in the new frontiers of bioremediation of recalcitrant materials such as plastics $[33,34]$. The presence of these enzymes could also be very interesting in food, pharmaceutical, detergent industry and in waste-water treatment. The production of enzymes with low optimum temperature by Antarctic fungi allows to exploit them in processing of thermolabile food products or in bioremediation of cold water [35-37].

A wide screening of soil yeasts from South Shetland archipelago and Antarctic Peninsula resulted in the isolation of strains belonging to 11 genera, among which Cryptococcus was the most represented. All the strains showed a wide enzymatic competence, with lipase, alkaline phosphatase and invertase activities being observed in most isolates. Among the isolates Cryptococcus gastricus (syn. Goffeauzyma gastrica), C. victoriae (syn. Vishniacozyma victoriae), C. gilvescens (syn. Goffeauzyma gilvencens), Leucosporidium sp., and Rhodotorula mucilaginosa showed toxicity against other yeasts via the secretion of a protein factor, being the first report of anti-yeast activity in Cryptococcus and Rhodotorula species [38].

A high number of predominately psychrotolerant yeasts isolated from soil (and water) samples collected at King George Island, as Mrakia and Cryptococcus species, Sporidiobolus salmonicolor, Leuconeurospora sp., Dioszegia fristingensis, and the most ubiquitous Rhodotorula laryngis (syn. Cystobasidium larynges) and 
Cr. victoriae, showed extracellular activities, as lipase, amylase and esterase, while chitinase and xylanase were less common [39].

The psychrotrophic, dimorphic yeast Candida humicola (syn. Vanrija humicola), isolated from Antarctic soil, was able to secrete an acidic protease, depending on the composition of the medium. Yeast nitrogen base medium depleted of amino acids or ammonium sulphate and supplemented with proteins gave higher enzyme activity. The purified protease had a molecular mass of 36,000 Da and was inhibited by pepstatin, iodoacetamide, and sodium dodecyl sulfate. It exhibited activity over a broad range of $\mathrm{pH}$ values ( $\mathrm{pH} 1$ to 7). During exponential growth the secretion was found to be greater at low than at higher temperatures. Despite that, the enzyme was active at temperatures ranging from 0 to $45^{\circ} \mathrm{C}$, with an optimum at $37^{\circ} \mathrm{C}$ [40]. This is of particular interest for the utilization of this enzyme, which may be produced at low temperatures and then used in combination with other enzymes extracted from mesophilic organisms.

A cold-adapted lipase was purified from the Antarctic filamentous fungus Geomyces sp. P7, isolated from soils collected in the neighborhood of the Arctowski Polish Antarctic Station at King George Island (Southern Shetlands). Its coding sequence was identified, cloned, and showed to be heterologously expressed in Saccharomyces cerevisiae BJ5465, retaining the same cold-adaption and thermostability characteristics of the native protein [41]. The most prominent producer of two different lipases, lipase A (CALA) and B (CALB), is an Antarctic strain of Candida antarctica (syn. Moesziomyces antarcticus), an alkali-tolerant yeast not found in soils, but in the sediment of Lake Vanda. Lipase B is probably the mostly employed hydrolase in the biocatalysts field, as an alternative to catalysts commonly used, whose residues in biomedical products are highly dangerous for human body [42].

Enzymes produced by Antarctic fungi may lead to important advancements in therapeutic applications. This is the case of 30 fungal strains isolated from soils (and mosses) of the Schirmacher Hills region (Dronning Maud Land, East Antarctica) identified as Aspergillus sp. IBBLA3, Coprinopsis cinerea IBBLA4, Coprinopsis sp. IBBLA5, Trichosporon asahii IBBLA1 and Aspergillus niger IBBLA2. They demonstrated to produce L-Asparaginase free of glutaminase and urease, proven to be competent in treating acute lymphoblastic leukaemia which is most common type of childhood cancer. Among the strains screened, T. asahii IBBLA1 exhibited the highest enzyme activity [43].

Antarctic fungi from soils of Livingstone Island in Maritime Antarctica demonstrated to be good producers of antioxidant enzymes, such as superoxide dismutase (SOD) and catalase (CAT), to survive oxidative stress, with potential application in medical and cosmetic industries. The statistically significant higher activity at 15 than at $30^{\circ} \mathrm{C}$ of identified SOD suggested that this cold-active enzyme likely helps fungi in maintaining oxidant-antioxidant balance at low temperature by scavenging superoxide radicals [44].

Some strains have demonstrated promising applications in bioremediation, including the breakdown of petroleum hydrocarbons. Petroleum contamination causes extensive damage in both Arctic and Antarctica, as ecosystem recovery is slower in cold than in warm regions. In 2001, between 1 and 10 million cubic meters of soil were considered to be contaminated in Antarctica [45]. The resistance of both filamentous fungi and yeasts from soils of Adelaide Island, Antarctic Peninsula, to aromatic and aliphatic hydrocarbons was demonstrated; this research lead to the hypothesis that Mortierella species may be able to use dodecane as carbon and energy source [46]. Subsequently, also a number of yeasts and filamentous fungi isolated from Macquarie Island contaminated soils showed to be able to degrade hydrocarbons [10]. Finally, a Penicillium CHY-2 strain, isolated from an unspecified Antarctic soil produced a manganese peroxidase $(\mathrm{MnP})$ degrading eight different aliphatic and aromatic hydrocarbons at low temperature conditions. This $\mathrm{MnP}$ consisted of monomers with a molecular mass of $36 \mathrm{kDa}$. The purified MnP had an optimum $\mathrm{pH}$ of 5.0 and temperature of $30^{\circ} \mathrm{C}$. The $\mathrm{Km}$ and Vmax values of $\mathrm{MnP}$ towards $\mathrm{Mn}^{2+}$ were $1.31 \mu \mathrm{M}$ and $185.19 \mu \mathrm{M} \mathrm{min}^{-1}$ respectively. These results indicated the strain CHY-2 as possible candidate to remove hydrocarbons in contaminated polar soils [47].

A powerful tool to analyze enzymes production in fungi is represented by proteomics. Despite proteomics applications on many strains of interest in different field is increasing in the last years, 
the only report for Antarctic fungi is the proteomic characterization of the black meristematic fungus Cryomyces antarcticus that lead to the identification of many enzymatic proteins [48]. The characterization of molecular competences of this organism and possibly of other related taxa is of great interest due to its extreme resistance to environmental stresses, proved also in the exposition to ionizing radiations and space conditions (see for example [49,50]).

Table 1. List of enzymes produced by Antarctic soil fungi, with their isolation site and the temperature at which the activity has been tested. Abbreviations: PG1, polygalacturonase; PG2, polypectate; PEC, pectinase; AM, amylase; CEL, cellulase; $\mathrm{CHI}$, chitinase; $\mathrm{PHO}$, phosphatase; GOD, glucose oxidase; URE, urease; PRO, protease; LIP, lipases; RNA, RNAase; DNA, DNAase; EST, esterase; AP, alkalin-phosphatase; INV, invertase; GEL, gelatinase; XYL, xylanase; ASP, asparaginase; SOD, superoxide dismutase; $\mathrm{CAT}$, catalase; MP, manganese peroxidase; KER, keratinase.

\begin{tabular}{|c|c|c|c|c|}
\hline $\begin{array}{l}\text { Enzyme } \\
\text { Category }\end{array}$ & Enzyme & Fungal Taxa & Collection Site & Temperature \\
\hline \multirow{19}{*}{$\begin{array}{l}\text { Carbohydrate } \\
\text { metabolism }\end{array}$} & \multirow{8}{*}{ PG1 } & $\begin{array}{l}\text { Akanthomyces lecanii (sub. } \\
\text { Verticillium lecanii sp. 2) [22] }\end{array}$ & Mt Melbourne & \multirow{8}{*}{$25^{\circ} \mathrm{C}$} \\
\hline & & Aspergillus versicolor [22] & Lamplugh Island & \\
\hline & & Chaetomium sp. [22] & Mt Melbourne & \\
\hline & & Cladosporium cladosporioides [22] & Crater Cirque & \\
\hline & & Dendryphiella salina [22] & $\begin{array}{l}\text { Lake "Carezza", } \\
\text { Baker Rocks }\end{array}$ & \\
\hline & & Phoma sorghina [22] & Kay Island & \\
\hline & & Phoma sp. [22] & $\begin{array}{l}\text { Lake “Carezza”, } \\
\text { Gondwana Station, } \\
\text { Crater Cirque }\end{array}$ & \\
\hline & & $\begin{array}{l}\text { Pseudogymnoascus pannorum (sub. } \\
\text { Geomyces pannorum) [22] }\end{array}$ & $\begin{array}{l}\text { Kay Island, Lake } \\
\text { "Carezza" }\end{array}$ & \\
\hline & \multirow{6}{*}{ PG2 } & Alternaria sp. [22] & Cape Washington & \multirow{6}{*}{$25^{\circ} \mathrm{C}$} \\
\hline & & Chaetomium sp. [22] & Mt Melbourne & \\
\hline & & Cladosporium cladosporioides [22] & Crater Cirque & \\
\hline & & C. herbarum [22] & $\begin{array}{c}\text { Crater Cirque, } \\
\text { Whitmer Peninsula }\end{array}$ & \\
\hline & & Phoma sorghina [22] & Kay Island & \\
\hline & & $\begin{array}{l}\text { Pseudogymnoascus pannorum (sub. } \\
\text { Geomyces pannorum) [22] }\end{array}$ & Lake "Carezza" & \\
\hline & \multirow{5}{*}{ PEC } & Alternaria sp. [22] & Cape Washington & \multirow{2}{*}{$25^{\circ} \mathrm{C}$} \\
\hline & & Aspergillus versicolor [22] & Lamplugh Island & \\
\hline & & Chaetomium sp. [22] & Mt Melbourne & $25,35^{\circ} \mathrm{C}$ \\
\hline & & Cladosporium herbarum [22] & $\begin{array}{c}\text { Crater Cirque, } \\
\text { Whitmer Peninsula }\end{array}$ & $25^{\circ} \mathrm{C}$ \\
\hline & & Cryptococcus sp. [38] & Deception Island & $10{ }^{\circ} \mathrm{C}$ \\
\hline
\end{tabular}


Table 1. Cont.

\begin{tabular}{|c|c|c|c|c|}
\hline $\begin{array}{l}\text { Enzyme } \\
\text { Category }\end{array}$ & Enzyme & Fungal Taxa & Collection Site & Temperature \\
\hline \multirow{26}{*}{$\begin{array}{l}\text { Carbohydrate } \\
\text { metabolism }\end{array}$} & & $\begin{array}{l}\text { Cystobasidium laryngis (sub. } \\
\text { Rhodotorula laryngis) [39] }\end{array}$ & \multirow{3}{*}{ King George Island } & $30{ }^{\circ} \mathrm{C}$ \\
\hline & & Dioszegia fristingensis [39] & & $22{ }^{\circ} \mathrm{C}$ \\
\hline & & Dioszegia sp. [39] & & $15^{\circ} \mathrm{C}$ \\
\hline & & Fellozyma sp. [38] & Snow Island & $22{ }^{\circ} \mathrm{C}$ \\
\hline & & $\begin{array}{l}\text { Goffeauzyma gilvescens (sub. } \\
\text { Cryptococcus gilvescens) [38] }\end{array}$ & Deception Island & $10{ }^{\circ} \mathrm{C}$ \\
\hline & & Kriegeria sp. [38] & Litchfield Island & $30{ }^{\circ} \mathrm{C}$ \\
\hline & & Leuconeurospora sp. $[31,39]$ & \multirow{5}{*}{ King George Island } & $\begin{array}{l}20^{\circ} \mathrm{C}[31] \\
15^{\circ} \mathrm{C}[39]\end{array}$ \\
\hline & & $\begin{array}{l}\text { Leucosporidium creatinivorum (sub. } \\
\text { Leucosporidiella creatinivora) [31] }\end{array}$ & & $4{ }^{\circ} \mathrm{C}$ \\
\hline & & $\begin{array}{l}\text { L. fragarium (sub. Leucosporidiella } \\
\text { fragaria) }[39]\end{array}$ & & $22{ }^{\circ} \mathrm{C}$ \\
\hline & & $\begin{array}{l}\text { L. muscorum (sub. Leucosporidiella } \\
\text { muscorum) [31] }\end{array}$ & & $4,20^{\circ} \mathrm{C}$ \\
\hline & & L. scottii [31] & & $4,20^{\circ} \mathrm{C}$ \\
\hline & & Leucosporidium sp. [38] & Litchfield Island & $15^{\circ} \mathrm{C}$ \\
\hline & & Metschnikowia sp. [39] & King George Island & $10^{\circ} \mathrm{C}$ \\
\hline & & Mrakia frigida (sub. M. gelida) [38] & Litchfield Island & $10{ }^{\circ} \mathrm{C}$ \\
\hline & & M. psychrophila [39] & \multirow{4}{*}{ King George Island } & $10^{\circ} \mathrm{C}$ \\
\hline & & M. robertii [39] & & $15^{\circ} \mathrm{C}$ \\
\hline & & $\begin{array}{l}\text { Naganishia antarctica (sub. } \\
\text { Cryptococcus antarcticus) [31] }\end{array}$ & & $20{ }^{\circ} \mathrm{C}$ \\
\hline & & $\begin{array}{l}\text { Phenoliferia glacialis (sub. } \\
\text { Rhodotorula glacialis) [39] }\end{array}$ & & $10,15^{\circ} \mathrm{C}$ \\
\hline & & Phoma sorghina [22] & Kay Island & \\
\hline & & Phoma sp. [22] & $\begin{array}{l}\text { Lake "Carezza", } \\
\text { Gondwana Station, } \\
\text { Crater Cirque }\end{array}$ & $25^{\circ} \mathrm{C}$ \\
\hline & & $\begin{array}{l}\text { Pseudogymnoascus pannorum (sub. } \\
\text { Geomyces pannorum) [22] }\end{array}$ & Lake “Carezza" & \\
\hline & & Sporidiobolus salmonicolor [39] & King George Island & $22{ }^{\circ} \mathrm{C}$ \\
\hline & \multirow{4}{*}{ AMY } & $\begin{array}{l}\text { Akanthomyces muscarius (sub. } \\
\text { Lecanicillium muscarium, det. as } \\
\text { Verticillium lecanii sp. 3) [22] }\end{array}$ & Kay Island & \multirow{4}{*}{$25^{\circ} \mathrm{C}$} \\
\hline & & $\begin{array}{l}\text { A. lecanii (sub. Verticillium lecanii } \\
\text { sp. 1, sp. 2) [22] }\end{array}$ & $\begin{array}{l}\text { Crater Cirque, Mt } \\
\text { Melbourne }\end{array}$ & \\
\hline & & Alternaria sp. [22] & Cape Washington & \\
\hline & & Aspergillus versicolor [22] & Lamplugh Island & \\
\hline
\end{tabular}


Table 1. Cont.

\begin{tabular}{|c|c|c|c|c|}
\hline $\begin{array}{l}\text { Enzyme } \\
\text { Category }\end{array}$ & Enzyme & Fungal Taxa & Collection Site & Temperature \\
\hline \multirow{24}{*}{$\begin{array}{l}\text { Carbohydrate } \\
\text { metabolism }\end{array}$} & \multirow{24}{*}{ AMY } & $\begin{array}{l}\text { Colletotrichum sp. } \\
\text { (sub. Glomerella sp.) [29] }\end{array}$ & King George Island & $25^{\circ} \mathrm{C}$ \\
\hline & & Cryptococcus sp. [38] & Deception Island & $10{ }^{\circ} \mathrm{C}$ \\
\hline & & Dendryphiella salina [22] & $\begin{array}{l}\text { Lake “Carezza”, } \\
\text { Baker Rocks }\end{array}$ & $25^{\circ} \mathrm{C}$ \\
\hline & & Dioszegia fristingensis [39] & \multirow{2}{*}{ King George Island } & $22{ }^{\circ} \mathrm{C}$ \\
\hline & & Dioszegia sp. [39] & & $15^{\circ} \mathrm{C}$ \\
\hline & & Fellozyma sp. [38] & Snow Island & $22{ }^{\circ} \mathrm{C}$ \\
\hline & & Geomyces spp. [29] & \multirow{3}{*}{ King George Island } & $4,25^{\circ} \mathrm{C}$ \\
\hline & & $\begin{array}{l}\text { Goffeauzyma gastrica (sub. } \\
\text { Cryptococcus gastricus) [39] }\end{array}$ & & $22{ }^{\circ} \mathrm{C}$ \\
\hline & & $\begin{array}{l}\text { G. gilvescens (sub. Cryptococcus } \\
\text { gilvescens) [39] }\end{array}$ & & $22{ }^{\circ} \mathrm{C}$ \\
\hline & & Hamamotoa sp. [38] & Dee Island & $4-10^{\circ} \mathrm{C}$ \\
\hline & & Holtermanniella wattica [39] & King George Island & $30{ }^{\circ} \mathrm{C}$ \\
\hline & & Hyphozyma sp. [38] & Nelson Island & $15^{\circ} \mathrm{C}$ \\
\hline & & Kriegeria sp. [38] & Litchfield Island & $30{ }^{\circ} \mathrm{C}$ \\
\hline & & Leuconeurospora sp. $[31,39]$ & King George Island & $\begin{array}{c}4,20^{\circ} \mathrm{C}[31] \\
15^{\circ} \mathrm{C}[39]\end{array}$ \\
\hline & & Mrakia blollopis [39] & & $15^{\circ} \mathrm{C}$ \\
\hline & & M. frigida (sub. M. gelida) [38,39] & $\begin{array}{l}\text { Litchfield Island } \\
\text { [38]; King George } \\
\text { Island [39] }\end{array}$ & $10{ }^{\circ} \mathrm{C}$ \\
\hline & & M. robertii [39] & & $15^{\circ} \mathrm{C}$ \\
\hline & & Mrakia sp. [39] & & $15^{\circ} \mathrm{C}$ \\
\hline & & $\begin{array}{l}\text { Naganishia antarctica (sub. } \\
\text { Cryptococcus antarcticus) [31] }\end{array}$ & & $4,20{ }^{\circ} \mathrm{C}$ \\
\hline & & $\begin{array}{l}\text { Phenoliferia glacialis (sub. } \\
\text { Rhodotorula glacialis) [39] }\end{array}$ & King George Island & $10,15^{\circ} \mathrm{C}$ \\
\hline & & Phialemonium sp. [29] & & $25^{\circ} \mathrm{C}$ \\
\hline & & Podospora sp. [29] & & $4,25^{\circ} \mathrm{C}$ \\
\hline & & Pseuderotium sp. [29] & & $4{ }^{\circ} \mathrm{C}$ \\
\hline & & $\begin{array}{l}\text { Pseudogymnoascus pannorum (sub. } \\
\text { Geomyces pannorum) [22] }\end{array}$ & $\begin{array}{l}\text { Kay Island, Lake } \\
\text { "Carezza", } \\
\text { "Giardino", } \\
\text { "Campo Icaro" }\end{array}$ & $25^{\circ} \mathrm{C}$ \\
\hline
\end{tabular}


Table 1. Cont.

\begin{tabular}{|c|c|c|c|c|}
\hline $\begin{array}{l}\text { Enzyme } \\
\text { Category }\end{array}$ & Enzyme & Fungal Taxa & Collection Site & Temperature \\
\hline \multirow{35}{*}{$\begin{array}{l}\text { Carbohydrate } \\
\text { metabolism }\end{array}$} & \multirow{5}{*}{ AMY } & Sporothrix inflata [29] & \multirow{3}{*}{ King George Island } & $25^{\circ} \mathrm{C}$ \\
\hline & & S. pallida [29] & & $25^{\circ} \mathrm{C}$ \\
\hline & & S. schenckii [29] & & $25^{\circ} \mathrm{C}$ \\
\hline & & $\begin{array}{c}\text { Vishniacozyma victoriae } \\
\text { (sub. Cryptococcus victoriae) [38] }\end{array}$ & Deception Island & $22{ }^{\circ} \mathrm{C}$ \\
\hline & & Wardomyces inflatus [29] & King George Island & $4{ }^{\circ} \mathrm{C}$ \\
\hline & \multirow{30}{*}{ CEL } & $\begin{array}{l}\text { Akanthomyces lecanii (sub. } \\
\text { Verticillium lecanii sp. 1) [22] }\end{array}$ & Crater Cirque & \\
\hline & & Alternaria sp. [22] & Cape Washington & $25^{\circ} \mathrm{C}$ \\
\hline & & Aspergillus versicolor [22] & Lamplugh Island & \\
\hline & & Candida glaebosa [31] & \multirow{3}{*}{ King George Island } & $4{ }^{\circ} \mathrm{C}$ \\
\hline & & C. sake [31] & & $4^{\circ} \mathrm{C}$ \\
\hline & & $\begin{array}{c}\text { Colletotrichum sp. } \\
\text { (sub. Glomerella sp.) [29] }\end{array}$ & & $25^{\circ} \mathrm{C}$ \\
\hline & & Cystobasidium sp. [38] & Dee Island & $15^{\circ} \mathrm{C}$ \\
\hline & & Debaryomyces hansenii [31] & King George Island & $4^{\circ} \mathrm{C}$ \\
\hline & & Dendryphiella salina [22] & Lake "Carezza" & $25^{\circ} \mathrm{C}$ \\
\hline & & Dioszegia fristingensis [39] & \multirow{2}{*}{ King George Island } & $22{ }^{\circ} \mathrm{C}$ \\
\hline & & Dioszegia sp. [31] & & $4{ }^{\circ} \mathrm{C}$ \\
\hline & & Fellozyma sp. [38] & Snow Island & $22{ }^{\circ} \mathrm{C}$ \\
\hline & & Filobasidium sp. [31] & \multirow{5}{*}{ King George Island } & $4,20^{\circ} \mathrm{C}$ \\
\hline & & Galerina fallax [29] & & $25^{\circ} \mathrm{C}$ \\
\hline & & Geomyces spp. [29] & & $4,25^{\circ} \mathrm{C}$ \\
\hline & & $\begin{array}{l}\text { Goffeauzyma gastrica (sub. } \\
\text { Cryptococcus gastricus) [39] }\end{array}$ & & $22{ }^{\circ} \mathrm{C}$ \\
\hline & & Holtermanniella wattica [39] & & $30{ }^{\circ} \mathrm{C}$ \\
\hline & & Hyphozyma sp. [38] & Nelson Island & $15^{\circ} \mathrm{C}$ \\
\hline & & Kriegeria sp. [38] & Litchfield Island & $30^{\circ} \mathrm{C}$ \\
\hline & & Leuconeurospora sp. [31,39] & \multirow{5}{*}{ King George Island } & $\begin{array}{l}4,20^{\circ} \mathrm{C}[31] ; \\
15^{\circ} \mathrm{C}[39]\end{array}$ \\
\hline & & $\begin{array}{l}\text { Leucosporidium creatinivorum (sub. } \\
\text { Leucosporidiella creatinivora) [31] }\end{array}$ & & $4,20^{\circ} \mathrm{C}$ \\
\hline & & $\begin{array}{l}\text { L. fragarium (sub. Leucosporidiella } \\
\text { fragaria) }[31,39]\end{array}$ & & $\begin{array}{l}4,20^{\circ} \mathrm{C}[31] ; \\
22{ }^{\circ} \mathrm{C}[39]\end{array}$ \\
\hline & & L. scottii [31] & & $4^{\circ} \mathrm{C}$ \\
\hline & & Mrakia blollopis [39] & & $15^{\circ} \mathrm{C}$ \\
\hline & & M. frigida (sub. M.gelida) [38,39] & $\begin{array}{l}\text { Litchfield Island } \\
\text { [35]; King George } \\
\text { Island [36] }\end{array}$ & $10{ }^{\circ} \mathrm{C}$ \\
\hline & & M. psychrophila [39] & \multirow{5}{*}{ King George Island } & $10^{\circ} \mathrm{C}$ \\
\hline & & M. robertii [39] & & $15^{\circ} \mathrm{C}$ \\
\hline & & Mrakia sp. [39] & & $15^{\circ} \mathrm{C}$ \\
\hline & & Nadsonia commutata [31] & & $4,20^{\circ} \mathrm{C}$ \\
\hline & & Phialemonium sp. [29] & & $25^{\circ} \mathrm{C}$ \\
\hline
\end{tabular}


Table 1. Cont.

\begin{tabular}{|c|c|c|c|c|}
\hline $\begin{array}{l}\text { Enzyme } \\
\text { Category }\end{array}$ & Enzyme & Fungal Taxa & Collection Site & Temperature \\
\hline \multirow{31}{*}{$\begin{array}{l}\text { Carbohydrate } \\
\text { metabolism }\end{array}$} & \multirow{12}{*}{ CEL } & Phoma sorghina [22] & Kay Island & \\
\hline & & Phoma sp. [22] & $\begin{array}{l}\text { Lake "Carezza", } \\
\text { Crater Cirque }\end{array}$ & $25^{\circ} \mathrm{C}$ \\
\hline & & Podospora sp. [29] & \multirow{2}{*}{ King George Island } & $4,25^{\circ} \mathrm{C}$ \\
\hline & & Pseuderotium sp. [29] & & $4{ }^{\circ} \mathrm{C}$ \\
\hline & & $\begin{array}{l}\text { Pseudogymnoascus pannorum } \\
\text { (sub. Geomyces pannorum) [22] }\end{array}$ & Lake “Carezza” & $25^{\circ} \mathrm{C}$ \\
\hline & & $\begin{array}{l}\text { Queenslandipenidiella kurandae } \\
\text { (sub. Penidiella kurandae) [29] }\end{array}$ & \multirow{4}{*}{ King George Island } & $25^{\circ} \mathrm{C}$ \\
\hline & & Sporothrix inflata [29] & & $25^{\circ} \mathrm{C}$ \\
\hline & & S. pallida [29] & & $25^{\circ} \mathrm{C}$ \\
\hline & & S. schenckii [29] & & $25^{\circ} \mathrm{C}$ \\
\hline & & Verticillium sp. [27] & $\begin{array}{l}\text { Great Wall Station, } \\
\text { King George Island }\end{array}$ & $38^{\circ} \mathrm{C}$ \\
\hline & & $\begin{array}{c}\text { Vishniacozyma victoriae (sub. } \\
\text { Cryptococcus victoriae) }[31,38,39]\end{array}$ & $\begin{array}{l}\text { King George Island } \\
\text { [31,39]; Deception } \\
\text { Island [38] }\end{array}$ & $\begin{array}{l}4,20^{\circ} \mathrm{C}[31] ; \\
22{ }^{\circ} \mathrm{C}[38] ; 15 \\
\quad{ }^{\circ} \mathrm{C}[39]\end{array}$ \\
\hline & & Wardomyces inflatus [29] & King George Island & $4{ }^{\circ} \mathrm{C}$ \\
\hline & \multirow{10}{*}{ INV } & Cryptococcus sp. [38] & Deception Island & $10^{\circ} \mathrm{C}$ \\
\hline & & Cystobasidium sp. [38] & Dee Island & $15^{\circ} \mathrm{C}$ \\
\hline & & Fellozyma sp. [38] & Snow Island & $22{ }^{\circ} \mathrm{C}$ \\
\hline & & $\begin{array}{l}\text { Goffeauzyma gilvescens (sub. } \\
\text { Cryptococcus gilvescens) [38] }\end{array}$ & Deception Island & $10^{\circ} \mathrm{C}$ \\
\hline & & Kriegeria sp. [38] & \multirow{3}{*}{ Litchfield Island } & $30^{\circ} \mathrm{C}$ \\
\hline & & Leucosporidium sp. [38] & & $15^{\circ} \mathrm{C}$ \\
\hline & & $\begin{array}{c}\text { Mrakia frigida } \\
\text { (sub. Mrakia gelida) [38] }\end{array}$ & & $10^{\circ} \mathrm{C}$ \\
\hline & & Rhodotorula mucilaginosa [38] & Snow Island & $30{ }^{\circ} \mathrm{C}$ \\
\hline & & Sporobolomyces roseus [38] & \multirow[b]{2}{*}{ Deception Island } & $22{ }^{\circ} \mathrm{C}$ \\
\hline & & $\begin{array}{l}\text { Vishniacozyma victoriae (sub. } \\
\text { Cryptococcus victoriae) [38] }\end{array}$ & & $22{ }^{\circ} \mathrm{C}$ \\
\hline & $X Y L$ & Dioszegia fristingensis [39] & King George Island & $22{ }^{\circ} \mathrm{C}$ \\
\hline & \multirow{8}{*}{$\mathrm{CHI}$} & $\begin{array}{l}\text { Akanthomyces muscarius (sub. } \\
\text { Lecanicillium muscarium, det. as } \\
\text { Verticillium lecanii sp. 3) }[22,23]\end{array}$ & Kay Island & $25{ }^{\circ} \mathrm{C}$ \\
\hline & & $\begin{array}{l}\text { A. lecanii (sub. Verticillium lecanii } \\
\text { sp. 1, sp. 2) [22] }\end{array}$ & $\begin{array}{l}\text { Crater Cirque, Mt } \\
\text { Melbourne }\end{array}$ & \\
\hline & & Dioszegia fristingensis [39] & \multirow{4}{*}{ King George Island } & $22{ }^{\circ} \mathrm{C}$ \\
\hline & & Leuconeurospora sp. [39] & & $15^{\circ} \mathrm{C}$ \\
\hline & & Metschnikowia sp. [39] & & $10^{\circ} \mathrm{C}$ \\
\hline & & Mrakia psychrophila [39] & & $10{ }^{\circ} \mathrm{C}$ \\
\hline & & $\begin{array}{l}\text { Pseudogymnoascus pannorum (sub. } \\
\text { Geomyces pannorum) [22] }\end{array}$ & $\begin{array}{l}\text { Kay Island, Lake } \\
\text { "Carezza", } \\
\text { "Giardino", } \\
\text { "Campo Icaro" }\end{array}$ & $25^{\circ} \mathrm{C}$ \\
\hline & & Sporidiobolus salmonicolor [39] & King George Island & $22{ }^{\circ} \mathrm{C}$ \\
\hline
\end{tabular}


Table 1. Cont.

\begin{tabular}{|c|c|c|c|c|}
\hline $\begin{array}{l}\text { Enzyme } \\
\text { Category }\end{array}$ & Enzyme & Fungal Taxa & Collection Site & Temperature \\
\hline $\begin{array}{l}\text { Carbohydrate } \\
\text { metabolism }\end{array}$ & GOD & $\begin{array}{l}\text { Pseudogymnoascus pannorum (sub. } \\
\text { Geomyces pannorum) [22] }\end{array}$ & $\begin{array}{l}\text { Lake "Carezza", } \\
\text { "Campo Icaro" }\end{array}$ & $25^{\circ} \mathrm{C}$ \\
\hline \multirow{27}{*}{$\begin{array}{l}\text { Protein } \\
\text { metabolism }\end{array}$} & \multirow{12}{*}{ URE } & Alternaria sp. [22] & Cape Washington & \multirow{2}{*}{$25^{\circ} \mathrm{C}$} \\
\hline & & Cladosporium cladosporioides [22] & Crater Cirque & \\
\hline & & Cystobasidium pallidum [38] & Deception Island & $30{ }^{\circ} \mathrm{C}$ \\
\hline & & Cystobasidium sp. [38] & Dee Island & $15^{\circ} \mathrm{C}$ \\
\hline & & Dendryphiella salina [22] & Lake "Carezza" & $25^{\circ} \mathrm{C}$ \\
\hline & & $\begin{array}{l}\text { Goffeauzyma gilvescens (sub. } \\
\text { Cryptococcus gilvescens) [38] }\end{array}$ & Deception Island & $10{ }^{\circ} \mathrm{C}$ \\
\hline & & Hamamotoa sp. [38] & Dee Island & $4,10^{\circ} \mathrm{C}$ \\
\hline & & Hyphozyma sp. [38] & Nelson Island & $15^{\circ} \mathrm{C}$ \\
\hline & & $\begin{array}{l}\text { Pseudogymnoascus pannorum } \\
\text { (sub. Geomyces pannorum) [22] }\end{array}$ & $\begin{array}{l}\text { Kay Island, Lake } \\
\text { "Carezza", } \\
\text { "Giardino", } \\
\text { "Campo Icaro" }\end{array}$ & $15,25^{\circ} \mathrm{C}$ \\
\hline & & Rhodotorula mucilaginosa [38] & Snow Island & $30^{\circ} \mathrm{C}$ \\
\hline & & Sporobolomyces roseus [38] & \multirow[b]{2}{*}{ Deception Island } & $22{ }^{\circ} \mathrm{C}$ \\
\hline & & $\begin{array}{l}\text { Vishniacozyma victoriae (sub. } \\
\text { Cryptococcus victoriae) [38] }\end{array}$ & & $22{ }^{\circ} \mathrm{C}$ \\
\hline & \multirow{15}{*}{ PRO } & $\begin{array}{l}\text { Akanthomyces muscarius (sub. } \\
\text { Lecanicillium muscarium, det. as } \\
\text { Verticillium lecanii sp. 3) [22] }\end{array}$ & Kay Island & $20^{\circ} \mathrm{C}$ \\
\hline & & $\begin{array}{c}\text { A. lecanii } \\
\text { (sub. Verticillium lecanii sp. 1) [22] }\end{array}$ & Crater Cirque & \\
\hline & & C. herbarum [22] & Whitmer Peninsula & \\
\hline & & Cryptococcus sp. [39] & \multirow{9}{*}{ King George Island } & $22{ }^{\circ} \mathrm{C}$ \\
\hline & & $\begin{array}{l}\text { Goffeauzyma gilvescens (sub. } \\
\text { Cryptococcus gilvescens) [39] }\end{array}$ & & $22{ }^{\circ} \mathrm{C}$ \\
\hline & & Leuconeurospora sp. [31,39] & & $\begin{array}{l}4{ }^{\circ} \mathrm{C}[31] ; 15 \\
{ }^{\circ} \mathrm{C}[39]\end{array}$ \\
\hline & & $\begin{array}{l}\text { Leucosporidium creatinivorum (sub. } \\
\text { Leucosporidiella creatinivora) [31] }\end{array}$ & & $4,20^{\circ} \mathrm{C}$ \\
\hline & & $\begin{array}{l}\text { Leucosporidium fragarium (sub. } \\
\text { Leucosporidiella fragaria) [31] }\end{array}$ & & $4,20^{\circ} \mathrm{C}$ \\
\hline & & $\begin{array}{l}\text { Leucosporidium muscorum (sub. } \\
\text { Leucosporidiella muscorum) [31] }\end{array}$ & & $4,20^{\circ} \mathrm{C}$ \\
\hline & & Leucosporidium scottii [31] & & $4,20^{\circ} \mathrm{C}$ \\
\hline & & Mrakia frigida (sub. M. gelida) [39] & & $10{ }^{\circ} \mathrm{C}$ \\
\hline & & Nadsonia commutata [31] & & $4{ }^{\circ} \mathrm{C}$ \\
\hline & & Sporobolomyces roseus [38] & Deception Island & $22{ }^{\circ} \mathrm{C}$ \\
\hline & & Sporidiobolus salmonicolor [39] & King George Island & $22{ }^{\circ} \mathrm{C}$ \\
\hline & & $\begin{array}{c}\text { Vanrija humicola (sub. Candida } \\
\text { humicola) [40] }\end{array}$ & Schirmacher Oasis & $4{ }^{\circ} \mathrm{C}$ \\
\hline
\end{tabular}


Table 1. Cont.

\begin{tabular}{|c|c|c|c|c|}
\hline $\begin{array}{l}\text { Enzyme } \\
\text { Category }\end{array}$ & Enzyme & Fungal Taxa & Collection Site & Temperature \\
\hline \multirow{12}{*}{$\begin{array}{l}\text { Protein } \\
\text { metabolism }\end{array}$} & \multirow{7}{*}{ GEL } & $\begin{array}{l}\text { Cystobasidium laryngis (sub. } \\
\text { Rhodotorula laryngis) [38] }\end{array}$ & Litchfield Island & $15^{\circ} \mathrm{C}$ \\
\hline & & Cystobasidium sp. [38] & Dee Island & $15^{\circ} \mathrm{C}$ \\
\hline & & Fellozyma sp. [38] & Snow Island & $22{ }^{\circ} \mathrm{C}$ \\
\hline & & Kriegeria sp. [38] & \multirow{2}{*}{ Litchfield Island } & $30^{\circ} \mathrm{C}$ \\
\hline & & Mrakia frigida (sub. M. gelida) [38] & & $10{ }^{\circ} \mathrm{C}$ \\
\hline & & Rhodotorula mucilaginosa [38] & Snow Island & $30{ }^{\circ} \mathrm{C}$ \\
\hline & & Sporobolomyces roseus [38] & Deception Island & $22{ }^{\circ} \mathrm{C}$ \\
\hline & \multirow{5}{*}{ ASP } & Aspergillus niger [43] & \multirow{5}{*}{ Schirmacher Hills } & \multirow{5}{*}{$30^{\circ} \mathrm{C}$} \\
\hline & & Aspergillus sp. [43] & & \\
\hline & & Coprinopsis cinerea [43] & & \\
\hline & & Coprinopsis sp. [43] & & \\
\hline & & Trichosporon asahii [43] & & \\
\hline \multirow{19}{*}{$\begin{array}{l}\text { Lipid } \\
\text { metabolism }\end{array}$} & \multirow{19}{*}{ LIP } & $\begin{array}{l}\text { Akanthomyces muscarius (sub. } \\
\text { Lecanicillium muscarium, det. as } \\
\text { Verticillium lecanii sp. 3) [22] }\end{array}$ & Kay Island & \multirow{7}{*}{$25^{\circ} \mathrm{C}$} \\
\hline & & $\begin{array}{l}\text { A. lecanii (sub. Verticillium lecanii } \\
\text { sp. 1, sp. 2) [22] }\end{array}$ & $\begin{array}{l}\text { Crater Cirque, } \\
\text { Mount Melbourne }\end{array}$ & \\
\hline & & Alternaria sp. [22] & Cape Washington & \\
\hline & & Aspergillus versicolor [22] & Lamplugh Island & \\
\hline & & Chaetomium sp. [22] & Mt Melbourne & \\
\hline & & Cladosporium cladosporioides [22] & Crater Cirque & \\
\hline & & C. herbarum [22] & $\begin{array}{c}\text { Crater Cirque, } \\
\text { Whitmer Peninsula }\end{array}$ & \\
\hline & & Cryptococcus sp. $[38,39]$ & $\begin{array}{l}\text { Deception Island } \\
\text { [38]; King George } \\
\text { Island [39] }\end{array}$ & $10{ }^{\circ} \mathrm{C}$ \\
\hline & & $\begin{array}{l}\text { Cystobasidium laryngis (sub. } \\
\text { Rhodotorula laryngis) [38,39] }\end{array}$ & $\begin{array}{l}\text { Litchfield Island } \\
\text { [38]; King George } \\
\text { Island [39] }\end{array}$ & $\begin{array}{l}15^{\circ} \mathrm{C}[38] ; 30 \\
{ }^{\circ} \mathrm{C}[39]\end{array}$ \\
\hline & & C. pallidum [38] & Deception Island & $30^{\circ} \mathrm{C}$ \\
\hline & & Cystobasidium sp. [38] & Dee Island & $15^{\circ} \mathrm{C}$ \\
\hline & & Dendryphiella salina [22] & $\begin{array}{l}\text { Lake "Carezza", } \\
\text { Baker Rocks }\end{array}$ & $25^{\circ} \mathrm{C}, 28^{\circ} \mathrm{C}$ \\
\hline & & Dioszegia fristingensis [39] & King George Island & $22{ }^{\circ} \mathrm{C}$ \\
\hline & & Fellozyma sp. [38] & Snow Island & $22{ }^{\circ} \mathrm{C}$ \\
\hline & & Geomyces sp. [41] & \multirow{2}{*}{ King George Island } & $35^{\circ} \mathrm{C}$ \\
\hline & & $\begin{array}{l}\text { Goffeauzyma gastrica (sub. } \\
\text { Cryptococcus gastricus) [39] }\end{array}$ & & $22{ }^{\circ} \mathrm{C}$ \\
\hline & & $\begin{array}{l}\text { Goffeauzyma gilvescens (sub. } \\
\text { Cryptococcus gilvescens) }[38,39]\end{array}$ & $\begin{array}{l}\text { Deception Island } \\
\text { [38]; King George } \\
\text { Island [39] }\end{array}$ & $\begin{array}{l}10{ }^{\circ} \mathrm{C}[38] ; 22 \\
\quad{ }^{\circ} \mathrm{C}[39]\end{array}$ \\
\hline & & Hamamotoa sp. [38] & Dee Island & $4-10{ }^{\circ} \mathrm{C}$ \\
\hline & & Hyphozyma sp. [38] & Nelson Island & $15^{\circ} \mathrm{C}$ \\
\hline
\end{tabular}


Table 1. Cont.

\begin{tabular}{|c|c|c|c|c|}
\hline $\begin{array}{l}\text { Enzyme } \\
\text { Category }\end{array}$ & Enzyme & Fungal Taxa & Collection Site & Temperature \\
\hline \multirow{18}{*}{$\begin{array}{l}\text { Lipid } \\
\text { metabolism }\end{array}$} & \multirow{18}{*}{ LIP } & Kriegeria sp. [38] & Litchfield Island & $30{ }^{\circ} \mathrm{C}$ \\
\hline & & Leuconeurospora sp. [39] & \multirow{4}{*}{ King George Island } & $15^{\circ} \mathrm{C}$ \\
\hline & & $\begin{array}{l}\text { Leucosporidium creatinivorum (sub. } \\
\text { Leucosporidiella creatinivora) }[31,39]\end{array}$ & & $4{ }^{\circ} \mathrm{C}$ \\
\hline & & $\begin{array}{l}\text { Leucosporidium fragarium (sub. } \\
\text { Leucosporidiella fragaria) [39] }\end{array}$ & & $22{ }^{\circ} \mathrm{C}$ \\
\hline & & Leucosporidium scottii [31] & & $4,20^{\circ} \mathrm{C}$ \\
\hline & & Leucosporidium sp. [38] & Litchfield Island & $15^{\circ} \mathrm{C}$ \\
\hline & & Metschnikowia sp. [39] & King George Island & $10{ }^{\circ} \mathrm{C}$ \\
\hline & & $\begin{array}{c}\text { Mrakia frigida } \\
\text { (sub. M. gelida) }[38,39]\end{array}$ & $\begin{array}{l}\text { Litchfield Island } \\
\text { [38]; King George } \\
\text { Island [39] }\end{array}$ & $10^{\circ} \mathrm{C}$ \\
\hline & & Mrakia robertii [39] & \multirow{3}{*}{ King George Island } & $15^{\circ} \mathrm{C}$ \\
\hline & & Mrakia sp. [39] & & $15^{\circ} \mathrm{C}$ \\
\hline & & $\begin{array}{c}\text { Phenoliferia glacialis } \\
\text { (sub. Rhodotorula glacialis) [39] }\end{array}$ & & $10,15^{\circ} \mathrm{C}$ \\
\hline & & Phoma sorghina [22] & Kay Island & \multirow{3}{*}{$25^{\circ} \mathrm{C}$} \\
\hline & & Phoma sp. [22] & $\begin{array}{l}\text { Lake "Carezza", } \\
\text { Gondwana Station, } \\
\text { Crater Cirque }\end{array}$ & \\
\hline & & $\begin{array}{l}\text { Pseudogymnoascus pannorum } \\
\text { (sub. Geomyces pannorum) [22] }\end{array}$ & $\begin{array}{l}\text { Kay Island, Lake } \\
\text { "Carezza", } \\
\text { "Giardino", } \\
\text { "Campo Icaro" }\end{array}$ & \\
\hline & & Rhodotorula mucilaginosa [38] & Snow Island & $30{ }^{\circ} \mathrm{C}$ \\
\hline & & Sporobolomyces roseus [38] & Deception Island & $22{ }^{\circ} \mathrm{C}$ \\
\hline & & Sporidiobolus salmonicolor [39] & King George Island & $22{ }^{\circ} \mathrm{C}$ \\
\hline & & $\begin{array}{c}\text { Vishniacozyma victoriae (sub. } \\
\text { Cryptococcus victoriae) }[31,38,39]\end{array}$ & $\begin{array}{l}\text { King Gerge Island } \\
\text { [31,39]; Deception } \\
\text { Island [38] }\end{array}$ & $\begin{array}{c}4{ }^{\circ} \mathrm{C}[31] ; 22 \\
{ }^{\circ} \mathrm{C}[38] ; 15^{\circ} \mathrm{C} \\
{[39]}\end{array}$ \\
\hline \multirow{5}{*}{$\begin{array}{l}\text { Nucleic } \\
\text { acids } \\
\text { metabolism }\end{array}$} & \multirow{5}{*}{ DNA } & $\begin{array}{l}\text { Akanthomyces muscarius (sub. } \\
\text { Lecanicillium muscarium, det. as } \\
\text { Verticillium lecanii sp. 3) [22] }\end{array}$ & Kay Island & $25^{\circ} \mathrm{C}$ \\
\hline & & Chaetomium sp. [22] & Mt Melbourne & $35^{\circ} \mathrm{C}$ \\
\hline & & C. herbarum [22] & Whitmer Peninsula & \multirow{3}{*}{$25^{\circ} \mathrm{C}$} \\
\hline & & Dendryphiella salina [22] & Lake “Carezza” & \\
\hline & & Phoma sp. [22] & $\begin{array}{l}\text { Gondwana Station, } \\
\text { Crater Cirque }\end{array}$ & \\
\hline
\end{tabular}


Table 1. Cont.

\begin{tabular}{|c|c|c|c|c|}
\hline $\begin{array}{l}\text { Enzyme } \\
\text { Category }\end{array}$ & Enzyme & Fungal Taxa & Collection Site & Temperature \\
\hline \multirow{7}{*}{$\begin{array}{l}\text { Nucleic } \\
\text { acids } \\
\text { metabolism }\end{array}$} & \multirow{7}{*}{ RNA } & $\begin{array}{l}\text { Akanthomyces muscarius (sub. } \\
\text { Lecanicillium muscarium, det. as } \\
\text { Verticillium lecanii sp. 3) [22] }\end{array}$ & Kay Island & \multirow{7}{*}{$25^{\circ} \mathrm{C}$} \\
\hline & & Aspergillus versicolor [22] & Lamplugh Island & \\
\hline & & Chaetomium sp. [22] & Mt Melbourne & \\
\hline & & Cladosporium cladosporioides [22] & Crater Cirque & \\
\hline & & C. herbarum [22] & $\begin{array}{c}\text { Crater Cirque, } \\
\text { Whitmer Peninsula }\end{array}$ & \\
\hline & & Phoma sp. [22] & Lake "Carezza" & \\
\hline & & $\begin{array}{l}\text { Pseudogymnoascus pannorum (sub. } \\
\text { Geomyces pannorum) [22] }\end{array}$ & "Campo Icaro" & \\
\hline \multirow{27}{*}{$\begin{array}{l}\text { Antioxidant } \\
\text { enzymes }\end{array}$} & \multirow{15}{*}{ SOD } & Aspergillus glaucus [44] & \multirow{15}{*}{ Livingston Island } & $20,25^{\circ} \mathrm{C}$ \\
\hline & & Aspergillus spp. [44] & & $25^{\circ} \mathrm{C}$ \\
\hline & & Cladosporium cladosporioides [44] & & $15^{\circ} \mathrm{C}$ \\
\hline & & C. herbarum [44] & & $20{ }^{\circ} \mathrm{C}$ \\
\hline & & C. oxysporum [44] & & $15^{\circ} \mathrm{C}$ \\
\hline & & Epicoccum nigrum [44] & & $15,20^{\circ} \mathrm{C}$ \\
\hline & & Monodictys austrina [44] & & \\
\hline & & Penicillium aurantiogriseum [44] & & $20{ }^{\circ} \mathrm{C}$ \\
\hline & & P. dierckxii [44] & & \\
\hline & & P. italicum [44] & & $25^{\circ} \mathrm{C}$ \\
\hline & & P. olsonii [44] & & \multirow[b]{2}{*}{$20{ }^{\circ} \mathrm{C}$} \\
\hline & & P. waksmanii [44] & & \\
\hline & & Penicillium spp. [44] & & $15,20^{\circ} \mathrm{C}$ \\
\hline & & $\begin{array}{l}\text { Pseudogymnoascus pannorum (sub. } \\
\text { Geomyces pannorum) }\end{array}$ & & $20,25^{\circ} \mathrm{C}$ \\
\hline & & Rhizopus sp. [44] & & $25^{\circ} \mathrm{C}$ \\
\hline & \multirow{12}{*}{ CAT } & Aspergillus glaucus [44] & \multirow{12}{*}{ Livingston Island } & $20,25^{\circ} \mathrm{C}$ \\
\hline & & Aspergillus spp. [44] & & $25^{\circ} \mathrm{C}$ \\
\hline & & Cladosporium cladosporioides [44] & & $15^{\circ} \mathrm{C}$ \\
\hline & & C. herbarum [44] & & $20^{\circ} \mathrm{C}$ \\
\hline & & C. oxysporum [44] & & $15^{\circ} \mathrm{C}$ \\
\hline & & Epicoccum nigrum [44] & & $15,20{ }^{\circ} \mathrm{C}$ \\
\hline & & Monodictys austrina [44] & & $20^{\circ} \mathrm{C}$ \\
\hline & & Penicillium aurantiogriseum [44] & & $20{ }^{\circ} \mathrm{C}$ \\
\hline & & P. dierckxii [44] & & $20^{\circ} \mathrm{C}$ \\
\hline & & P. italicum [44] & & $25^{\circ} \mathrm{C}$ \\
\hline & & P. olsonii [44] & & \multirow{2}{*}{$20{ }^{\circ} \mathrm{C}$} \\
\hline & & P. waksmanii [44] & & \\
\hline
\end{tabular}


Table 1. Cont.

\begin{tabular}{|c|c|c|c|c|}
\hline $\begin{array}{l}\text { Enzyme } \\
\text { Category }\end{array}$ & Enzyme & Fungal Taxa & Collection Site & Temperature \\
\hline \multirow{4}{*}{$\begin{array}{l}\text { Antioxidant } \\
\text { enzymes }\end{array}$} & \multirow{3}{*}{ CAT } & Penicillium spp. [44] & & $15,20{ }^{\circ} \mathrm{C}$ \\
\hline & & $\begin{array}{l}\text { Pseudogymnoascus pannorum (sub. } \\
\text { Geomyces pannorum) [44] }\end{array}$ & & $20,25^{\circ} \mathrm{C}$ \\
\hline & & Rhizopus sp. [44] & & $25^{\circ} \mathrm{C}$ \\
\hline & MP & Penicillium sp. [47] & $\begin{array}{l}\text { Not specified } \\
\text { Antarctic site }\end{array}$ & $20{ }^{\circ} \mathrm{C}$ \\
\hline \multirow{26}{*}{$\begin{array}{l}\text { Other } \\
\text { hydrolithic } \\
\text { enzymes }\end{array}$} & \multirow{7}{*}{$\mathrm{PHO}$} & $\begin{array}{l}\text { Akanthomyces muscarius (sub. } \\
\text { Lecanicillium muscarium, det. as } \\
\text { Verticillium lecanii sp.3) [22] }\end{array}$ & Kay Island & \multirow{7}{*}{$25^{\circ} \mathrm{C}$} \\
\hline & & $\begin{array}{l}\text { A. lecanii (sub. Verticillium lecanii } \\
\text { sp. 1, sp. 2) [22] }\end{array}$ & $\begin{array}{c}\text { Crater Cirque, } \\
\text { Mount Melbourne }\end{array}$ & \\
\hline & & Aspergillus versicolor [22] & Lamplugh Island & \\
\hline & & Chaetomium sp. [22] & Mt Melbourne & \\
\hline & & C. herbarum [22] & $\begin{array}{c}\text { Crater Cirque, } \\
\text { Whitmer Peninsula }\end{array}$ & \\
\hline & & Phoma sorghina [22] & Kay Island & \\
\hline & & $\begin{array}{l}\text { Pseudogymnoascus pannorum (sub. } \\
\text { Geomyces pannorum) [22] }\end{array}$ & $\begin{array}{l}\text { "Giardino", } \\
\text { "Campo Icaro" }\end{array}$ & \\
\hline & \multirow{19}{*}{ EST } & Cryptococcus sp. [38] & Deception Island & $10{ }^{\circ} \mathrm{C}$ \\
\hline & & $\begin{array}{l}\text { Cystobasidium laryngis (sub. } \\
\text { Rhodotorula laryngis) }[38,39]\end{array}$ & $\begin{array}{l}\text { Litchfield Island } \\
\text { [38]; King George } \\
\text { Island [39] }\end{array}$ & $\begin{array}{c}15^{\circ} \mathrm{C}[38] ; 30 \\
{ }^{\circ} \mathrm{C}[39]\end{array}$ \\
\hline & & C. pallidum [38] & Deception Island & $30^{\circ} \mathrm{C}$ \\
\hline & & Dioszegia crocea [31] & \multirow{6}{*}{ King George Island } & $4,20^{\circ} \mathrm{C}$ \\
\hline & & D. fristingensis [39] & & $22{ }^{\circ} \mathrm{C}$ \\
\hline & & D. hungarica [31] & & $4,20^{\circ} \mathrm{C}$ \\
\hline & & Dioszegia sp. [31,39] & & $\begin{array}{l}4,20{ }^{\circ} \mathrm{C}[31] \\
15{ }^{\circ} \mathrm{C}[39]\end{array}$ \\
\hline & & Glaciozyma antarctica [39] & & $10^{\circ} \mathrm{C}$ \\
\hline & & $\begin{array}{l}\text { Goffeauzyma gastrica (sub. } \\
\text { Cryptococcus gastricus) [39] }\end{array}$ & & $22{ }^{\circ} \mathrm{C}$ \\
\hline & & $\begin{array}{l}\text { G. gilvescens (sub. Cryptococcus } \\
\text { gilvescens) [38] }\end{array}$ & Deception Island & $10^{\circ} \mathrm{C}$ \\
\hline & & Hyphozyma sp. [38] & Nelson Island & $15^{\circ} \mathrm{C}$ \\
\hline & & Leuconeurospora sp. [31,39] & \multirow{4}{*}{ King George Island } & $\begin{array}{l}4{ }^{\circ} \mathrm{C}[31] ; 15 \\
{ }^{\circ} \mathrm{C}[39]\end{array}$ \\
\hline & & $\begin{array}{l}\text { Leucosporidium creatinivorum (sub. } \\
\text { Leucosporidiella creatinivora) }[31,39]\end{array}$ & & $\begin{array}{c}4,20{ }^{\circ} \mathrm{C}[31] \\
22{ }^{\circ} \mathrm{C}[39]\end{array}$ \\
\hline & & $\begin{array}{l}\text { L. fragarium (sub. Leucosporidiella } \\
\text { fragaria) }[31,39]\end{array}$ & & $\begin{array}{c}4,20{ }^{\circ} \mathrm{C}[31] \\
22{ }^{\circ} \mathrm{C}[39]\end{array}$ \\
\hline & & L. scottii [31] & & $4,20^{\circ} \mathrm{C}$ \\
\hline & & Leucosporidium sp. [38] & Litchfield Island & $15^{\circ} \mathrm{C}$ \\
\hline & & Mrakia blollopis [39] & \multirow{3}{*}{ King George Island } & $15^{\circ} \mathrm{C}$ \\
\hline & & M. psychrophila [39] & & $10{ }^{\circ} \mathrm{C}$ \\
\hline & & Nadsonia commutata [31] & & $4,20^{\circ} \mathrm{C}$ \\
\hline
\end{tabular}


Table 1. Cont.

\begin{tabular}{|c|c|c|c|c|}
\hline $\begin{array}{l}\text { Enzyme } \\
\text { Category }\end{array}$ & Enzyme & Fungal Taxa & Collection Site & Temperature \\
\hline \multirow{16}{*}{$\begin{array}{l}\text { Other } \\
\text { hydrolithic } \\
\text { enzymes }\end{array}$} & \multirow{3}{*}{ EST } & $\begin{array}{l}\text { Phenoliferia glacialis (sub. } \\
\text { Rhodotorula glacialis) [39] }\end{array}$ & & $15^{\circ} \mathrm{C}$ \\
\hline & & Sporobolomyces roseus [38] & Deception Island & $22{ }^{\circ} \mathrm{C}$ \\
\hline & & $\begin{array}{c}\text { Vishniacozyma victoriae (sub. } \\
\text { Cryptococcus victoriae) }[31,38,39]\end{array}$ & $\begin{array}{l}\text { King George Island } \\
\text { [31,39]; Deception } \\
\text { Island [38] }\end{array}$ & $\begin{array}{l}4,20{ }^{\circ} \mathrm{C}[31] ; \\
22{ }^{\circ} \mathrm{C}[38] ; 15 \\
\quad{ }^{\circ} \mathrm{C}[39]\end{array}$ \\
\hline & \multirow{13}{*}{$\mathrm{AP}$} & Cryptococcus sp. [38] & Deception Island & $10{ }^{\circ} \mathrm{C}$ \\
\hline & & $\begin{array}{l}\text { Cystobasidium laryngis (sub. } \\
\text { Rhodotorula laryngis) [38] }\end{array}$ & Litchfield Island & $15^{\circ} \mathrm{C}$ \\
\hline & & Cystobasidium pallidum [38] & Deception Island & $30{ }^{\circ} \mathrm{C}$ \\
\hline & & Cystobasidium sp. [38] & Dee Island & $15^{\circ} \mathrm{C}$ \\
\hline & & Fellozyma sp. [38] & Snow Island & $22{ }^{\circ} \mathrm{C}$ \\
\hline & & $\begin{array}{l}\text { Goffeauzyma gilvescens (sub. } \\
\text { Cryptococcus gilvescens) [38] }\end{array}$ & Deception Island & $10{ }^{\circ} \mathrm{C}$ \\
\hline & & Hamamotoa sp. [38] & Dee Island & $4-10^{\circ} \mathrm{C}$ \\
\hline & & Kriegeria sp. [38] & \multirow{3}{*}{ Litchfield Island } & $30{ }^{\circ} \mathrm{C}$ \\
\hline & & Leucosporidium sp. [38] & & $15^{\circ} \mathrm{C}$ \\
\hline & & Mrakia frigida (sub. M. gelida) [38] & & $10{ }^{\circ} \mathrm{C}$ \\
\hline & & Rhodotorula mucilaginosa [38] & Snow Island & $30{ }^{\circ} \mathrm{C}$ \\
\hline & & Sporobolomyces roseus [38] & \multirow{2}{*}{ Deception Island } & \multirow[b]{2}{*}{$22{ }^{\circ} \mathrm{C}$} \\
\hline & & $\begin{array}{l}\text { Vishniacozyma victoriae (sub. } \\
\text { Cryptococcus victoriae) [38] }\end{array}$ & & \\
\hline
\end{tabular}

\section{Other Bioactive Compounds}

In addition to enzymes, fungi are a rich reservoir of different classes of secondary metabolites, such as terpenoids, polyketides, alkaloids, polyacetylenes with demonstrated antiviral, antibacterial, antifungal, antitumoral, herbicidal and antiprotozoal activities. These molecules play a pivotal role in the inter- and intra-specific interactions within the soil microbial communities and provide them competitive advantages over other microorganisms [11,51]. A list of bioactive compounds retrieved from Antarctic soil fungi, grouped according to their biological activity is reported in Table 2.

Because of the recent emergence of antibiotic-resistant pathogenic microorganisms, and the connected risks for public health, the search for novel classes of active compounds in this context is worth to be developed for its strong practical importance, as it has been recognized by the World Health Organization as a threat to human health [52,53]. Extreme environments could be an excellent source of new antibiotics and, in this context, Antarctica, an almost unknown continent, is potentially of great interest. Aspergillus and Penicillium species are well-known producers of many bioactive compounds. Penicillium species are likely among the most abundant and widespread in different environments and substrates and many of them demonstrated to produce different bioactive compounds $[54,55]$. An overview of secondary metabolites with versatile antimicrobial potential was reported by Bratchkova and Ivanova [56] from Arctic and Antarctic microorganisms and their possible role in the adaptation and survival of microorganisms in the ice deserts was discussed. A noticeable antimicrobial activity was registered by a $P$. nalgiovense strain, from a soil sample close to an abandoned penguin nest at Paulete Island (Maritime Antarctica). This fungal strain was able to secrete the antifungal secondary metabolite amphotericin B; this ability was noteworthy, since it was the first time that amphotericin B had been isolated from an organism other than the bacterium Streptomyces nodosus [57]. Different fungal 
strains isolated from Maritime Antarctic sites showed a significant inhibitory effect on pathogenic bacteria, but a weaker effect as antimycotic [58]. Antiviral activities against the H1N1 and H3N2 influenza viruses were demonstrated by a soil strain of Aspergillus ochraceopetaliformis [59], thanks to the presence of compounds such as ochraceopone A, isoasteltoxin, and asteltoxin.

Some strains are good producers of secondary metabolites with multiple activities. Godinho et al. [11] found multiple antiviral, antibacterial, antifungal, antitumoral, herbicidal and antiprotozoal activities in extracts of Aspergillus sydowii, Penicillium allii-sativi, P. brevicompactum, P. chrysogenum and P. rubens strains isolated from cold oligotrophic soils of the Union Glacier region in continental Antarctica. Particularly interesting were the findings of a broad high antiviral activity against Dengue virus 2, and antiprotozoal and antifungal activities by a strain of P. brevicompactum. The authors also observed that the same species may have different bioactivities and suggested that the low fungal diversity of the Antarctic oligotrophic soils may present a high intra-specific diversity [11]. Thus, as already stated above, it is mandatory to keep different isolates of the same species of fungi in the culture collections, to make them available for further studies.

Penicillium tardochrysogenum isolated from the McMurdo Dry Valleys was reported as a new endemic species of Antarctica, able to produce asperentins, penicillins, secalonic acids, and the partial characterised extrolite met $\varnothing$ [60].

In maritime Antarctica, some endophytic fungi isolated from healthy specimens of $D$. antarctica and $C$. quitensis, and others from the rhizosphere soil of $D$. antarctica, showed to produce bioactive molecules with antimicrobial, antiprotozoal and antitumoral activities [61,62]. Extracts of 19 out of 564 of the endophytic isolates showed leishmanicidal activity and 6 inhibited the growth of at least one tumor cell line [61]. Among the root inhabitants, the extract of Purpureocillium lilacinum exhibited high trypanocidal, antifungal, and antibacterial activities, with moderate toxicity for normal human cells [62]. Cytotoxic metabolites with biological activity against various cancer lines were also isolated from the psychrophilic fungus Oidiodendron truncatum GW3-13 [63] and numerous psychrotrophic fungi isolated from different substrates collected on the Fildes Peninsula (King George Island) [64]. These latter also showed antimicrobial activity, inhibiting the growth of different bacteria in vitro, with differences among isolates of the same species [65].

New molecules with potential interesting activities, produced by Antarctic fungi, were isolated and characterized. Indeed, one new alkaloid (acremolin C) and four known compounds with weak to moderate antibacterial activities were isolated from cultures of the soil fungus Aspergillus sydowii [66]. New metabolites (asterric acid derivatives) with antifungal activity against Aspergillus fumigatus and antimicrobial activities against Gram-positive and Gram-negative bacteria were extracted from cultures of a Geomyces sp. strain isolated from a soil sample collected at the Fildes Peninsula, King George Island [67]. Three new metabolites were obtained from an Antarctic soil-derived Penicillium sp., showing cytotoxicity against the human cell lines tested and antituberculosis activity [68].

Strains of G. pannorum, isolated from Maritime Antarctica, gave a stronger antioxidant response, evaluated on phenolic compounds production, after cold shock [44,69]. This was suggested as a mechanism to overcome the life-endangering influence of low temperature and survive intracellular cold-induced oxidative stress.

Great is the interest for the production of exopolysaccharides because their different applications in food, pharmaceutical, cosmetic, and other industries. Five psychrophilic exopolysaccharide producing yeast strains were isolated from Livingston Island: Cryptococcus laurentii (syn. Papiliotrema laurentii) AL65, Sporobolomyces salmonicolor AL36, Debaryomyces hansenii, Leucosporidium scottii and Rhodotorula glutinis. The highest exopolysaccharide yield was established for L. scottii, and significant differences in the metabolites profiling were observed using NMR spectroscopy, probably representing different adaptation strategies to Antarctic conditions, according to the phylogenetic position. Differences were observed in the production of some biotechnologically important metabolites like alanine, leucine and/or tyrosine, used for medical treatments. Some of these, along with extracellular polysaccharides, could be used in the practice as part of waste-free and more cost-effective technologies [70]. 
Cryptococcus is one of the most frequent yeast genera in this environment, with numerous new species described for the science, as Cr. vishniacii (syn. Naganishia vishniacii), Cr. friedmannii (syn. Naganishia friedmannii), Cr. antarcticus (syn. Naganishia antarctica), Cr. albidosimilis (syn. Naganishia albidosimilis) [71-73]. An isolate of Cr. laurentii (syn. Papiliotrema laurentii) from Antarctic soil, identified basing on its morphological, cultural and physiological properties, was selected as an active producer of exopolysaccharides, which resulted to be optimal emulsifiers with applications in cosmetic products [74].

Ocampo-Friedmann and Friedmann [75] proved a weak ability of nearly 40 cryptoendolithic fungal strains to produce biologically active substances inhibiting the growth of algae and cyanobacteria within the lichen-dominated cryptoendolithic communities of the McMurdo Dry Valleys. These substances were suggested to have a role in maintaining the zonation of microorganisms in the community.

The search for new forms of defense from plant diseases, less harmful to the environment, has nowadays become increasingly required. Thus, many works have dealt with yeasts and filamentous fungi from Antarctic soils to assess their bioactivity potential against specific or generic pathogens. Most of the fungi tested for this purpose have been isolated from maritime Antarctic soils. A number of filamentous fungi belonging to the genera Pseudogymnoascus and Cladosporium from Deception Island (South Shetland Islands, Antarctica) soils demonstrated to produce compounds against phytopathogens such as Xanthomonas species [76,77]. Bioactive metabolites against neglected tropical diseases and pests were reported also from the Antarctic Peninsula: Pseudogymnoascus destructans, Mortierella parvispora, and Penicillium chrysogenum displayed antiparasitic activities, whilst extracts of $P$. destructans, M. amoeboidea, Mortierella sp. 3, and P. tardochrysogenum showed herbicidal activities [78]. The production of promising biotechnological molecules by Mortierella species was not a novelty, as already reported for an endophytic M. alpina strain isolated from Schistidium antarctici, a moss species widely distributed at King George Island [79].

Among Antarctic microorganisms, yeasts have been mainly suggested as the best tool for promoting the control of several phytopathologies in agriculture, for fruits storage and/or transport in cold chambers, as an important alternative to synthetic chemical fungicides. Despite that, the work in this field is still limited [80]. An inhibitory effect on the growth or germination of pathogens may be due to metabolites generated by yeasts or the production of lytic enzymes. For example, a psychrotrophic isolate of Leucosporidium scottii from Antarctic soils, producing soluble and volatile antifungal substances inhibiting the growth of pathogens on apples, was identified as a good biocontrol agent [81]. Moreover, two isolates of Candida sake, from soil and water samples collected in King Gorge Island, were reported as biocontrol agents in apples stored at low temperature, as producer of antifungal volatile organic compounds (VOCs) which inhibited the growth of five apple pathogens (P. expansum, Botrytis cinerea, A. alternata, A. tenuissima, and A. arborescens) [82].

Finally, some Antarctic fungal taxa exhibited a considerable melanins production. The characterization of these pigments is of great interest, due to their scavenging, radio- and photo-protective, antimicrobial, cytotoxic, anti-inflammatory, and immunomodulatory activities. As an example, their study in the Antarctic black fungus Cryomyces antarcticus [83] led to very interesting results in the frame of the biotechnological applications [84]. 
Table 2. Bioactive compounds produced by soil fungi, grouped according to their activity. Fungal taxa and target organisms or tumoral cell lines are reported. Biological activities without a detected chemical compound are reported as "not analyzed". Compounds not expressing a biological activity are reported as "checked activity not significant". Compounds not checked for their activity are reported as "not evaluated". Further details about the isolation locality of each strain, the temperature at which the activities were tested and other notes are available in Table S1.

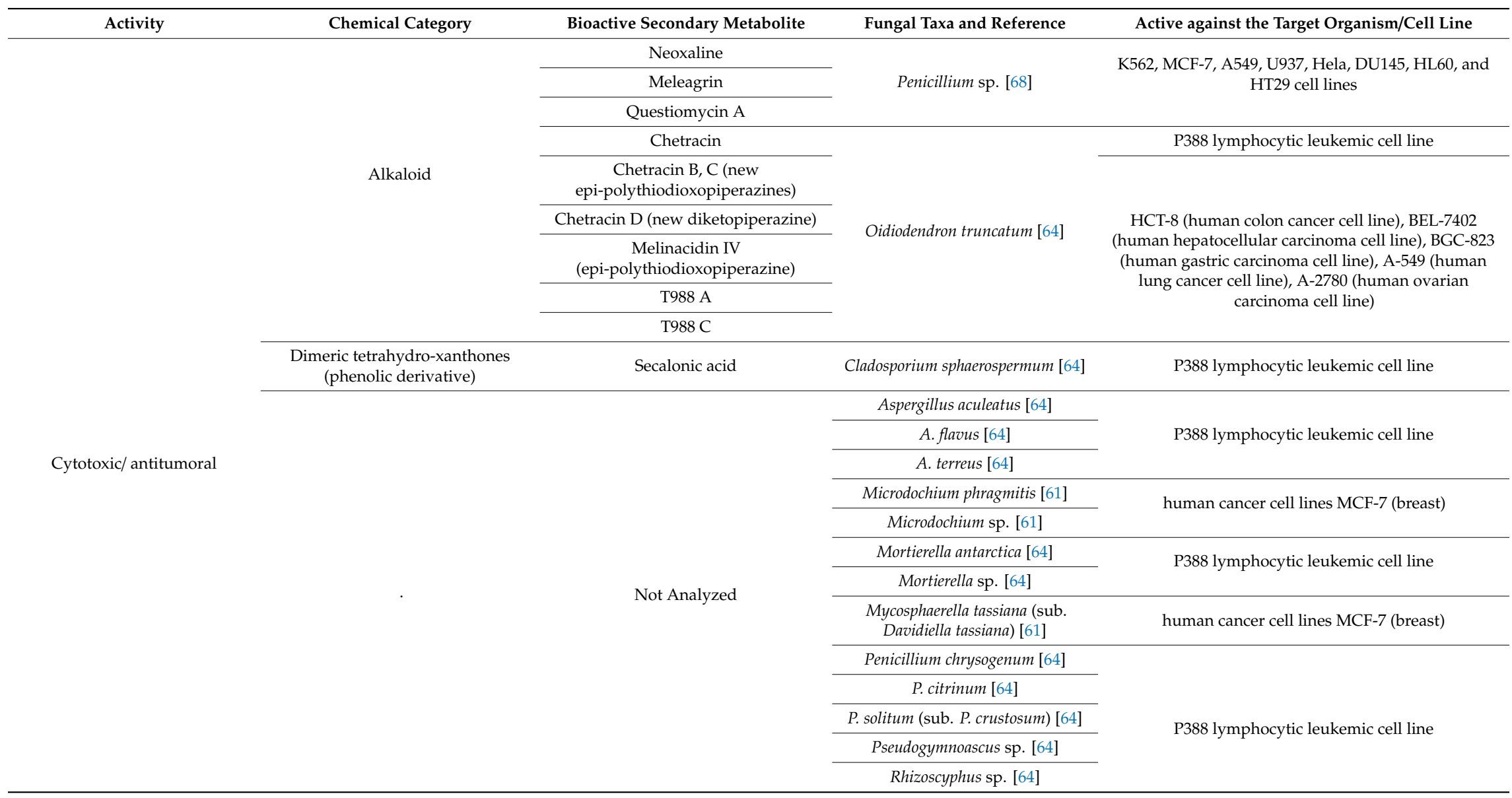


Table 2. Cont

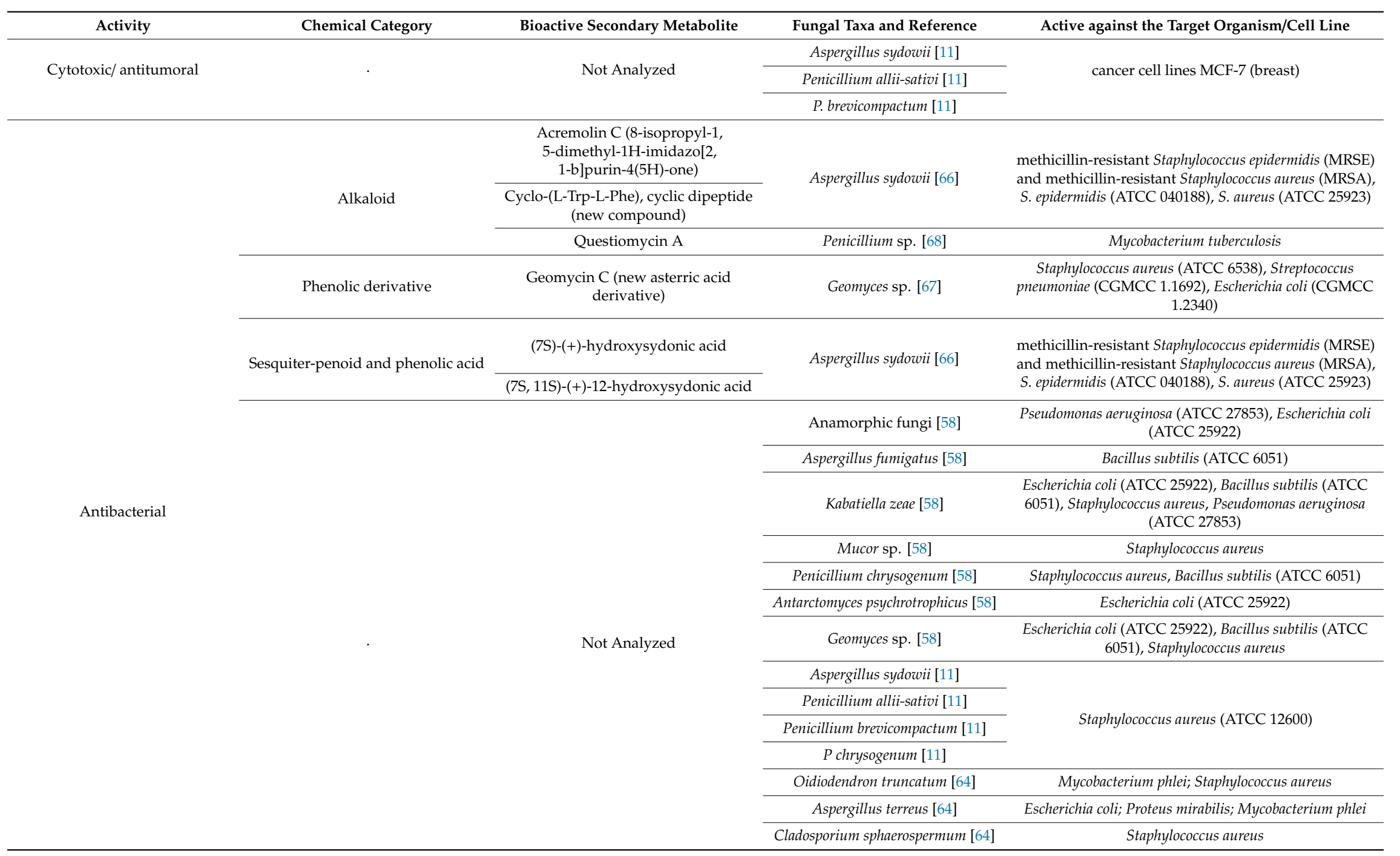


Table 2. Cont.

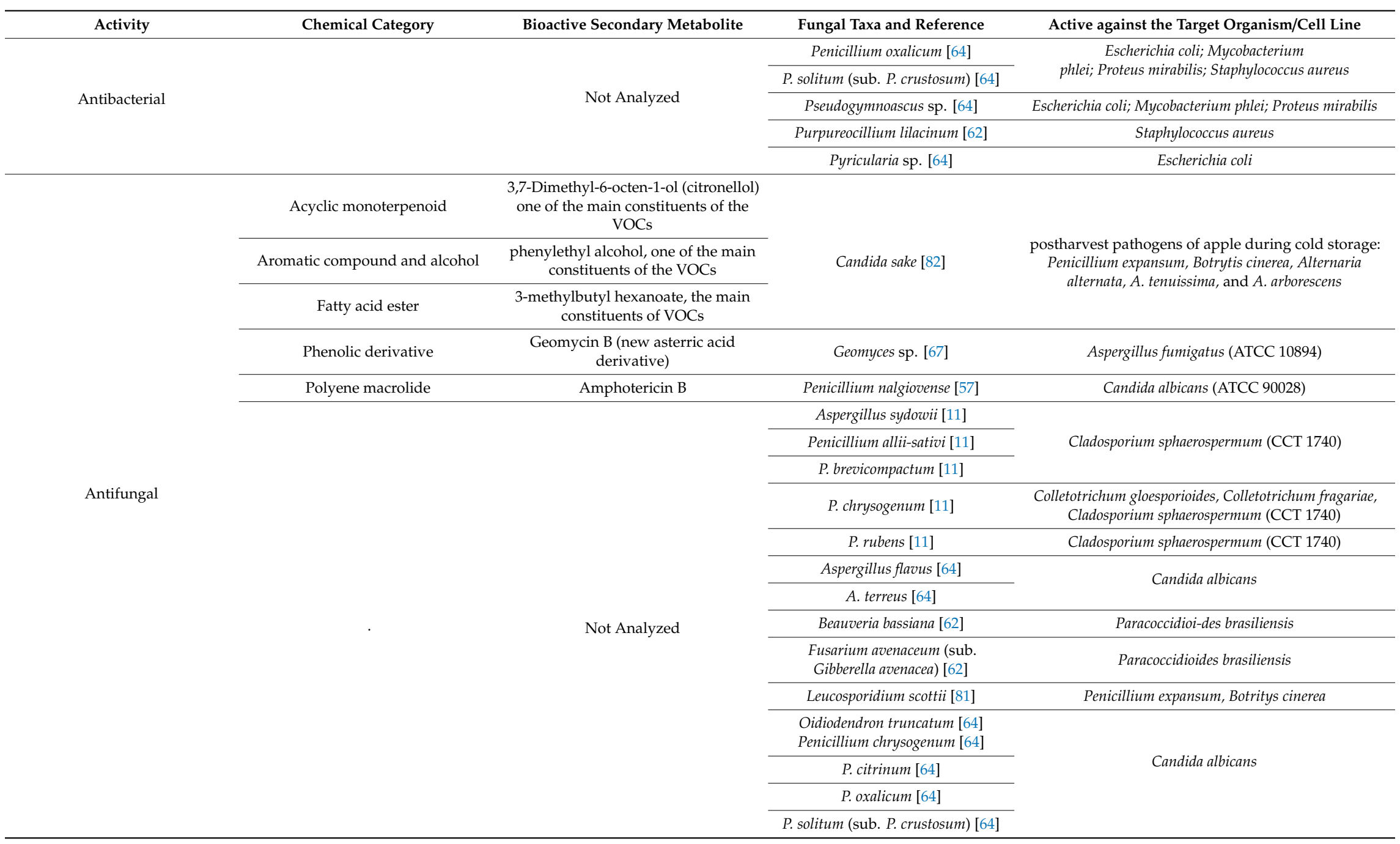


Table 2. Cont.

\begin{tabular}{|c|c|c|c|c|}
\hline Activity & Chemical Category & Bioactive Secondary Metabolite & Fungal Taxa and Reference & Active against the Target Organism/Cell Line \\
\hline \multirow{11}{*}{ Antifungal } & \multirow{11}{*}{. } & \multirow{11}{*}{ Not Analyzed } & Penicillium sp. [62] & \multirow{11}{*}{ Paracoccidioides brasiliensis } \\
\hline & & & Peniophora sp. [62] & \\
\hline & & & Pestalotiopsis microspora [62] & \\
\hline & & & Phanerochaete sp. [62] & \\
\hline & & & Pseudeurotium sp. [62] & \\
\hline & & & Pseudogymnoascus sp. [62] & \\
\hline & & & Purpureocillium lilacinum [62] & \\
\hline & & & Schizophyllum commune [62] & \\
\hline & & & Simplicillium lamellicola [62] & \\
\hline & & & Trichoderma longibrachiatum [62] & \\
\hline & & & Trichosporon asteroides [62] & \\
\hline \multirow{14}{*}{ Anti-protozoal } & \multirow{14}{*}{. } & \multirow{14}{*}{ Not Analyzed } & Penicillium brevicompactum [11] & Trypanosoma cruzi \\
\hline & & & Alternaria sp. [61] & \multirow{8}{*}{ Leishmania amazonensis (strain IFLA/BR/196/PH-8 } \\
\hline & & & Antarctomyces psychrotrophicus [61] & \\
\hline & & & Cadophora luteo-olivacea [61] & \\
\hline & & & Helgardia sp. [61] & \\
\hline & & & Herpotrichia sp. [61] & \\
\hline & & & Oculimacula sp. [61] & \\
\hline & & & Phaeosphaeria herpotrichoides [61] & \\
\hline & & & Phaeosphaeria sp. [61] & \\
\hline & & & Purpureocillium lilacinum [62] & Trypanosoma cruzi \\
\hline & & & Mortierella amoeboidea [78] & Leishmania amazonensis (strain IFLA/BR/196/PH-8) \\
\hline & & & M. parvispora $[78]$ & $\begin{array}{c}\text { Trypanosoma cruzi, Leishmania amazonensis (strain } \\
\text { IFLA/BR/196/PH-8) }\end{array}$ \\
\hline & & & Penicillium chrysogenum [78] & \\
\hline & & & Pseudogymnoascus destructans [78] & Leishmania amazonensis (strain IFLA/BR/196/PH-8) \\
\hline
\end{tabular}


Table 2. Cont.

\begin{tabular}{|c|c|c|c|c|}
\hline Activity & Chemical Category & Bioactive Secondary Metabolite & Fungal Taxa and Reference & Active against the Target Organism/Cell Line \\
\hline \multirow{6}{*}{ Antiviral } & \multirow{2}{*}{ Furofuran-triene $\alpha$-pyrone } & Asteltoxin & \multirow{3}{*}{$\begin{array}{l}\text { Aspergillus ochraceopetali-formis } \\
{[59]}\end{array}$} & \multirow{3}{*}{$\mathrm{H} 1 \mathrm{~N} 1$ and $\mathrm{H} 3 \mathrm{~N} 2$ influenza viruses } \\
\hline & & Isoasteltoxin & & \\
\hline & $\alpha$-Pyrone merosesqui-terpenoid & Ochraceopone A & & \\
\hline & & \multirow{3}{*}{ Not Analyzed } & Penicillium allii-sativi [11] & \multirow{3}{*}{ Dengue virus 2} \\
\hline & & & P. brevicompactum [11] & \\
\hline & & & P. chrysogenum [11] & \\
\hline \multirow{4}{*}{ Herbicidal } & & \multirow{4}{*}{ Not Analyzed } & Penicillium chrysogenum [11] & \multirow{3}{*}{$\begin{array}{c}\text { Lactuca sativa (lettuce) and Agrostis stolonifera } \\
\text { (bentgrass) }\end{array}$} \\
\hline & & & Mortierella sp. [78] & \\
\hline & & & Penicillium tardochrysogenum [78] & \\
\hline & & & Pseudogymnoascus destructans [78] & Agrostis stolonifera (bentgrass) \\
\hline \multirow{8}{*}{ Checked activity not significant } & \multirow{8}{*}{ Alkaloid } & Chetoseminudin C & Oidiodendron truncatum [63] & \\
\hline & & $\begin{array}{c}(E)-3-(1 H \text {-imidazole-4- } \\
\text { ylmethylene)-6-(1H-indl-3- } \\
\text { ylmethyl)-2,5-piperazinediol }\end{array}$ & Penicillium sp. [68] & \\
\hline & & $\begin{array}{l}\text { Isopenilline A (new indolyl } \\
\text { diketopiperazine derivative) }\end{array}$ & Penicillium sp. [68] & \\
\hline & & $\begin{array}{c}\text { Penilline A (new indolyl } \\
\text { diketopiperazine derivative) }\end{array}$ & \multirow{3}{*}{ Penicillium sp. [68] } & \\
\hline & & $\begin{array}{c}\text { Penilline B (new indolyl } \\
\text { diketopiperazine derivative) }\end{array}$ & & \\
\hline & & Penilloid A (new compound) & & \\
\hline & & cyclo-L-Trp-Lser (cyclic dipeptid) & \multirow{2}{*}{ Oidiodendron truncatum [63] } & \\
\hline & & T988 B & & \\
\hline
\end{tabular}


Table 2. Cont.

\begin{tabular}{|c|c|c|c|c|}
\hline Activity & Chemical Category & Bioactive Secondary Metabolite & Fungal Taxa and Reference & Active against the Target Organism/Cell Line \\
\hline \multirow{8}{*}{ Checked activity not significant } & Carboxylic acid and phenol & 4-hydroxyphenylacetic acid & Aspergillus sydowii [66] & \\
\hline & Phenolic derivative and ester & Ethyl asterrate (new diphenyl ether) & \multirow{6}{*}{ Geomyces sp. [67] } & \\
\hline & Phenolic derivative and ester & $n$-butyl asterrate (new diphenyl ether) & & \\
\hline & Phenolic derivative & asterric acid & & \\
\hline & Phenolic derivative and acid & Methyl asterrate & & \\
\hline & Phenolic derivative and acid & $\begin{array}{c}\text { Geomycin A (new asterric acid } \\
\text { derivative) }\end{array}$ & & \\
\hline & $\begin{array}{l}\text { Phenolic derivative (spiro } \\
\text { compound) }\end{array}$ & Bisdechloro-geodin & & \\
\hline & Merosesquiterpenoid & $\begin{array}{l}\text { ochraceopone } \mathrm{B}, \mathrm{C}, \mathrm{D}, \mathrm{E} \text { (new highly } \\
\text { oxygenated } \alpha \text {-pyrone } \\
\text { merosesquiter-penoids) }\end{array}$ & Aspergillus ochraceopetaliformis [59] & \\
\hline \multirow{18}{*}{ Activity not evaluated } & $\gamma$-Amino acid & $\gamma$-Aminobutyric acid (GABA) & Debaryomyces hansenii [70] & \\
\hline & Beta-lactam derivatives & Penicillins & Penicillium tardochrysogenum [60] & \\
\hline & Carboxylic acid amide & $\mathrm{N}$-(2-hydroxyphenyl)- acetamide & Penicillium sp. [68] & \\
\hline & \multirow{3}{*}{ Carboxylic acid } & Acetic acid & \multirow{2}{*}{ Debaryomyces hansenii [70] } & \\
\hline & & Formic acid & & \\
\hline & & & Rhodotorula glutinis [70] & \\
\hline & $\begin{array}{c}\text { Dimeric tetrahydro-xanthones } \\
\text { (phenolic derivative) }\end{array}$ & Secalonic acid D, F & Penicillium tardochrysogenum [60] & \\
\hline & \multirow{3}{*}{ Exopolysaccharides } & \multirow{3}{*}{ Exopolysaccharides } & Cryptococcus laurentii AL62 [85] & \\
\hline & & & Debaryomyces hansenii [70] & \\
\hline & & & Rhodotorula glutinis [70] & \\
\hline & Heterocyclic compound & 2-Benzoxazolinone & Penicillium sp. [68] & \\
\hline & Isocoumarins (phenolic derivatives) & Asperentins & P. tardochryso-genum [60] & \\
\hline & \multirow{5}{*}{ Primary alchool } & \multirow{5}{*}{ Choline } & Cryptococcus laurentii [70] & \\
\hline & & & Debaryomyces hansenii [70] & \\
\hline & & & Leucosporidium scotii [70] & \\
\hline & & & Rhodotorula glutinis [70] & \\
\hline & & & Sporobolomyces salmonicolor [70] & \\
\hline & & partially characterized 'met $\varnothing$ ' & Penicillium tardochrysogenum [60] & \\
\hline
\end{tabular}




\section{Conclusions}

In 1991, a landmark paper estimated that there are 1.5 million fungi on the Earth [86]. Molecular methods have dramatically increased our knowledge on this kingdom. Subsequent estimations suggested that as many as 5.1 million fungal species exist, of which only a small percentage was known (about 10\%), while the majority remained to be discovered [87]. Based on more recently generated data from culture-dependent and -independent surveys, the fungal species on the Earth have been estimated to be 12 (11.7-13.2) million at least, and an even higher diversity than this estimation is expected [88]. Therefore, fungi are an unmapped and untapped source of enzymes and secondary metabolites with an incredible application potential. Unknown fungal species and their constituent metabolites can be identified by investigating the microbiota of soil or biological samples from extreme or unexplored environments. The undiscovered microbial diversity of cold environments is today threatened by climate change. The risk of dissemination of non-indigenous microorganisms into indigenous microbial communities as consequence of climate change has been forecasted. This could result in changes of the composition of soil communities and even the disappearance of species of potential interest. Moreover, climate change that has been affecting the maritime Antarctic region for a longer time respect to the rest of the continent, concerns a real risk of dispersal of potentially pathogenic species by wild birds, which can fly great distances towards South America and Oceania. This is the case reported for some fungi potentially pathogenic to humans, isolated from ornithogenic soils of the Antarctic Peninsula, where they possibly live in a latent state [89].

It is common knowledge that extreme environmental conditions in Antarctica exerted an important selective pressure for many species of photosynthetic organisms, fauna and microorganisms to evolve unique characteristics and capabilities which could be used for biotechnological development. The growing commercial interest in Antarctic research raises key policy, ethical and moral questions, addressed in a report by Lohan and Johnston [90], with the inputs of many researchers, where the absence of clear rules governing the use of genetic resources from Antarctica was clearly reported. Although the products or processes based on Antarctic organisms have not been yet commercialized, bioprospecting has been deeply and carefully debated within the international research community and the Antarctic Treaty Consultative Meeting (ATCM). Despite years of discussion, there has been little progress on using the Antarctic Treaty System to regulate bioprospecting activities. As a consequence, the only resolution adopted was that of the 28th ATCM held in 2005 at Stockholm, Sweden, calling on the Treaty Parties to remind their national Antarctic programs and other research institutes engaged in Antarctic biological prospecting activities of the provisions of Article III.1 of the Treaty, concerning scientific exchanges and the availability of scientific observations and results from Antarctica (the Secretariat of the Antarctic Treaty, https://www.ats.aq/e/secretariat.html).

Supplementary Materials: The following is available online at http://www.mdpi.com/1660-4601/17/18/6459/s1, Table S1: Bioactive compounds produced from soil fungi, grouped according to their biological activity. Names of fungal taxa, isolation sites, temperature and target organisms or tumoral cell lines are reported. Biological activities without a detected chemical compound are reported as "not analyzed". Compounds not expressing a biological activity are reported as "checked activity not significant". Compounds not checked for their biological activity are reported as "not evaluated". Notes refer to strains isolation or growth temperatures.

Author Contributions: Conceptualization, L.Z.; methodology, resources, data curation, L.Z., S.T., M.E.T., F.C.; writing-original draft preparation, L.Z., F.C.; writing-review and editing, L.Z., S.T., M.E.T., F.C.; project administration, funding acquisition, S.T. and L.Z. All authors have read and agreed to the published version of the manuscript.

Funding: This research was funded by the Italian National Program for Antarctic Researches (PNRA14 000_132 and PNRA18_00015).

Acknowledgments: The authors wish to thank the Italian National Program for Antarctic Researches for funding research activities in Italy in the frame of Italian Antarctic Research Program (PNRA) projects. The authors wish to thank Giovanni Vidari, retired full professor of the Department of Chemistry, University of Pavia, for his precious supervision of the Table 2. 
Conflicts of Interest: The authors declare no conflict of interest. The funders had no role in the design of the study; in the collection, analyses, or interpretation of data; in the writing of the manuscript, or in the decision to publish the results.

\section{References}

1. Øvstedal, D.O.; Lewis Smith, R.I. Lichens of Antarctica and South Georgia; Cambridge University Press: Cambridge, UK, 2001; p. 424.

2. Friedmann, E.I. Antarctic Microbiology; Wiley-Liss: New York, NY, USA, 1993; p. 634.

3. Ruisi, S.; Barreca, D.; Selbmann, L.; Zucconi, L.; Onofri, S. Fungi in Antarctica. Rev. Environ. Sci. Biotechnol. 2007, 6, 127-141. [CrossRef]

4. Onofri, S.; Zucconi, L.; Tosi, S. Continental Antarctic Fungi; IHW-Verlag: Eching, Germany, 2007; p. 247.

5. Durán, P.; Barra, P.J.; Jorquera, M.A.; Viscardi, S.; Fernandez, C.; Paz, C.; Mora, M.L.; Bol, R. Occurrence of Soil Fungi in Antarctic Pristine Environments. Front. Bioeng. Biotechnol. 2019, 7, 28. [CrossRef]

6. Bridge, P.D.; Spooner, B.M. Non-lichenized Antarctic fungi: Transient visitors or members of a cryptic ecosystem? Fungal Ecol. 2012, 5, 381-394. [CrossRef]

7. Rosa, L.H.; Zani, C.L.; Cantrell, C.L.; Duke, S.O.; Van Dijck, P.; Desideri, A.; Rosa, C.A. Fungi in Antarctica: Diversity, ecology, effects of climate change, and bioprospection for bioactive compounds. In Fungi of Antarctica. Diversity, Ecology and Biotechnological Application; Rosa, L.H., Ed.; Springer Nature: Basel, Switzerland, 2019; pp. 1-17. ISBN 978-3-030-18366-0.

8. Kirby, B.M.; Barnard, D.; Marla Tuffin, I.; Cowan, D.A. Ecological Distribution of Microorganisms in Terrestrial, Psychrophilic Habitats. In Extremophiles Handbook; Horikoshi, K., Ed.; Springer: Tokyo, Japan, 2011; pp. 839-863.

9. Lambrechts, S.; Willems, A.; Tahon, G. Uncovering the uncultivated majority in antarctic soils: Toward a synergistic approach. Front. Microbiol. 2019, 10, 242. [CrossRef]

10. Ferrari, B.C.; Zhang, C.; Van Dorst, J. Recovering greater fungal diversity from pristine and diesel fuel contaminated sub-Antarctic soil through cultivation using both a high and a low nutrient media approach. Front. Microbiol. 2011, 2, 217. [CrossRef]

11. Godinho, V.M.; Gonçalves, V.N.; Santiago, I.F.; Figueredo, H.M.; Vitoreli, G.A.; Schaefer, C.E.; Barbosa, E.C.; Oliveira, J.G.; Alves, T.M.A.; Zani, C.L.; et al. Diversity and bioprospection of fungal community present in oligotrophic soil of continental Antarctica. Extremophiles 2015, 19, 585-596. [CrossRef]

12. Duarte, A.W.F.; dos Santos, J.A.; Vianna, M.V.; Vieira, J.M.F.; Mallagutti, V.H.; Inforsato, F.J.; Wentzel, L.C.P.; Lario, L.D.; Rodrigues, A.; Pagnocca, F.C.; et al. Cold-adapted enzymes produced by fungi from terrestrial and marine Antarctic environments. Crit. Rev. Biotechnol. 2018, 38, 600-619. [CrossRef] [PubMed]

13. De Pascale, D.; De Santi, C.; Fu, J.; Landfald, B. The microbial diversity of Polar environments is a fertile ground for bioprospecting. Mar. Genom. 2012, 8, 15-22. [CrossRef] [PubMed]

14. Chan, Y.; Van Nostrand, J.D.; Zhou, J.; Pointing, S.B.; Farrell, R.L. Functional ecology of an Antarctic dry valley. Proc. Natl. Acad. Sci. USA 2013, 110, 8990-8995. [CrossRef] [PubMed]

15. Fierer, N.; Leff, J.W.; Adams, B.J.; Nielsen, U.N.; Bates, S.T.; Lauber, C.L.; Owens, S.; Gilbert, J.A.; Wall, D.H.; Caporaso, J.G. Cross-biome metagenomic analyses of soil microbial communities and their functional attributes. Proc. Natl. Acad. Sci. USA 2012, 109, 21390-21395. [CrossRef]

16. Dennis, P.; Rushton, S.; Newsham, K.; Laudicina, V.; Ord, V.; Daniell, T.; O’Donnell, A.G.; Hopkins, D.W. Soil fungal community composition does not alter along a latitudinal gradient through the maritime and sub-Antarctic. Fungal Ecol. 2012, 5, 403-408. [CrossRef]

17. Canini, F.; Geml, J.; D'Acqui, L.P.; Selbmann, L.; Onofri, S.; Ventura, S.; Zucconi, L. Exchangeable cations and $\mathrm{pH}$ drive diversity and functionality of fungal communities in biological soil crusts from coastal sites of Victoria Land, Antarctica. Fungal Ecol. 2020, 45, 100923. [CrossRef]

18. Marx, J.C.; Collins, T.; D'Amico, S.; Feller, G.; Gerday, C. Cold-adapted enzymes from marine Antarctic microorganisms. Mar. Biotechnol. 2007, 9, 293-304. [CrossRef] [PubMed]

19. Feller, G.; Gerday, C. Psychrophilic enzymes: Hot topics in cold adaptation. Nat. Rev. Microbiol. 2003, 1, 200-208. [CrossRef] [PubMed]

20. Cavicchioli, R.; Siddiqui, K.S.; Andrews, D.; Sowers, K.R. Low-temperature extremophiles and their applications. Curr. Opin. Biotechnol. 2002, 13, 253-261. [CrossRef] 
21. Martorell, M.M.; Ruberto, L.A.M.; de Figueroa, L.I.C.; Mac Cormack, W.P. Antarctic Yeasts as a Source of Enzymes for Biotechnological Applications. In Fungi of Antarctica. Diversity, Ecology and Biotechnological Application; Rosa, L.H., Ed.; Springer Nature: Basel, Switzerland, 2019; pp. 285-304. ISBN 978-3-030-18366-0.

22. Fenice, M.; Selbmann, L.; Zucconi, L.; Onofri, S. Production of extracellular enzymes by Antarctic fungal strains. Polar Biol. 1997, 17, 275-280. [CrossRef]

23. Fenice, M. The psychrotolerant Antarctic fungus Lecanicillium muscarium CCFEE 5003: A powerful producer of cold-tolerant chitinolytic enzymes. Molecules 2016, 21, 447. [CrossRef]

24. Onofri, S.; Fenice, M.; Cicalini, A.R.; Tosi, S.; Magrino, A.; Pagano, S.; Selbmann, L.; Zucconi, L.; Vishniac, H.S.; Ocampo-Friedmann, R.; et al. Ecology and biology of microfungi from Antarctic rocks and soils. Ital. J. Zool. 2000, 67, 163-168. [CrossRef]

25. Fenice, M.; Selbmann, L.; Di Giambattista, R.; Federici, F. Chitinolytic activity at low temperature of an Antarctic strain (A3) of Verticillium lecanii. Res. Micobiol. 1998, 149, 289-300. [CrossRef]

26. Hamid, R.; Khan, M.A.; Ahmad, M.; Ahmad, M.M.; Abdin, M.Z.; Musarrat, J.; Javed, S. Chitinases: An update. J. Pharm. Bioall. Sci. 2013, 5, 21-29. [CrossRef]

27. Wang, N.; Zang, J.; Ming, K.; Liu, Y.; Wu, Z.; Ding, H. Production of cold-adapted cellulase by Verticillium sp. isolated from Antarctic soils. Electron. J. Biotechnol. 2013, 16, 10. [CrossRef]

28. Wentzel, L.C.P.; Inforsato, F.J.; Montoya, Q.V.; Rossin, B.G.; Nascimento, N.R.; Rodrigues, A.; Sette, L.D. Fungi from Admiralty Bay (King George Island, Antarctica) soils and marine sediments. Microb. Ecol. 2019, 77, 12-24. [CrossRef] [PubMed]

29. Krishnan, A.; Convey, P.; Gonzalez-Rocha, G.; Alias, S.A. Production of extracellular hydrolase enzymes by fungi from King George Island. Polar Biol. 2016, 39, 65-76. [CrossRef]

30. Hopkins, D.W.; Sparrow, A.D.; Shillam, L.L.; English, L.C.; Dennis, P.G.; Novis, P.; Elberling, B.; Gregorich, E.G.; Greenfield, L.G. Enzymatic activities and microbial communities in an Antarctic dry valley soil: Responses to $C$ and N supplementation. Soil Biol. Biochem. 2008, 40, 2130-2136. [CrossRef]

31. Vaz, A.B.; Rosa, L.H.; Vieira, M.L.; Garcia, V.D.; Brandão, L.R.; Teixeira, L.C.; Moliné, M.; Libkind, D.; von Broock, M.; Rosa, C.A. The diversity, extracellular enzymatic activities and photoprotective compounds of yeasts isolated in Antarctica. Braz. J. Microbiol. 2011, 42, 937-947. [CrossRef]

32. Panda, T.; Gowrishankar, B.S. Production and application of esterases. Appl. Microbiol. Biotechnol. 2005, 67, 160-169. [CrossRef]

33. Magnin, A.; Pollet, E.; Perrin, R.; Ullmann, C.; Persillon, C.; Phalip, V.; Avérous, L. Enzymatic recycling of thermoplastic polyurethanes: Synergistic effect of an esterase and an amidase and recovery of building blocks. Waste Manag. 2019, 85, 141-150. [CrossRef]

34. Tahir, L.; Ali, M.I.; Zia, M.; Atiq, M.; Hasan, F.; Ahmed, S. Production and Characterization of Esterase in Lantinus tigrinus for Degradation of Polystyrene. Pol. J. Microbiol. 2013, 62, 101-108. [CrossRef]

35. McKelvey, S.M.; Murphy, R.A. Biotechnological Use of Fungal Enzymes. In Fungi Biology and Applications; Kavanagh, K., Ed.; John Wiley \& Sons, Inc.: Hoboken, NJ, USA, 2017; pp. 201-225. [CrossRef]

36. Rani, K.; Datt, S.; Rana, R. Brief review on alkaline phosphatases- an overview. Int. J. Microbiol. Bioinf. 2012, $2,1-4$.

37. Kulshrestha, S.; Tyagi, P.; Sindhi, V.; Yadavilli, K.S. Invertase and its applications-A brief review. J. Pharm. Res. 2013, 7, 792-797. [CrossRef]

38. Troncoso, E.; Barahona, S.; Carrasco, M.; Villarreal, P.; Alcaíno, J.; Cifuentes, V.; Baeza, M. Identification and characterization of yeasts isolated from the South Shetland Islands and the Antarctic Peninsula. Polar Biol. 2016, 40, 649-658. [CrossRef]

39. Carrasco, M.; Rozas, J.M.; Barahona, S.; Alcaíno, J.; Cifuentes, V.; Baeza, M. Diversity and extracellular enzymatic activities of yeasts isolated from King George Island, the sub-Antarctic region. BMC Microbiol. 2012, 12, 251. [CrossRef] [PubMed]

40. Ray, M.K.; Devi, K.U.; Kumar, G.S.; Shivaji, S. Extracellular protease from the antarctic yeast Candida humicola. Appl. Environ. Microbiol. 1992, 58, 1918-1923. [CrossRef]

41. Florczak, T.; Daroch, M.; Wilkinson, M.C.; Białkowska, A.; Bates, A.D.; Turkiewicz, M.; Iwanejko, L.A. Purification, characterization and expression in Saccharomyces cerevisiae of LipG7 an enantioselective, cold-adapted lipase from the Antarctic filamentous fungus Geomyces sp. P7 with unusual thermostability characteristics. Enzym. Microb. Technol. 2013, 53, 18-24. [CrossRef] [PubMed] 
42. Kundys, A.; Białecka-Florjańczyk, E.; Fabiszewska, A.; Małajowicz, J. Candida antarctica lipase B as catalyst for cyclic esters synthesis, their polymerization and degradation of aliphatic polyesters. J. Polym. Environ. 2018, 26, 396-407. [CrossRef]

43. Ashok, A.; Doriya, K.; Rao, J.V.; Qureshi, A.; Tiwari, A.K.; Kumar, D.S. Microbes Producing L-asparaginase free of glutaminase and urease isolated from extreme locations of antarctic soil and moss. Sci. Rep. 2019, 9, 1423. [CrossRef] [PubMed]

44. Tosi, S.; Kostadinova, N.; Krumova, E.; Pashova, S.; Dishliiska, V.; Spassova, B.; Vassilev, S.; Angelova, M. Antioxidant enzyme activity of filamentous fungi isolated from Livingston Island, Maritime Antarctica. Polar Bol. 2010, 33, 1227-1237. [CrossRef]

45. Snape, I.; Riddle, M.J.; Stark, J.S.; Cole, C.M.; King, C.K.; Duquesne, S.; Gore, D. Management and remediation of contaminated sites at Casey Station, Antarctica. Polar Rec. 2001, 37, 199-214. [CrossRef]

46. Hughes, K.A.; Bridge, P.; Clark, M.S. Tolerance of Antarctic soil fungi to hydrocarbons. Sci. Total Environ. 2007, 372, 539-548. [CrossRef]

47. Govarthanan, M.; Fuzisawa, S.; Hosogai, T.; Chang, Y.C. Biodegradation of aliphatic and aromatic hydrocarbons using the filamentous fungus Penicillium sp. CHY-2 and characterization of its manganese peroxidase activity. RSC Adv. 2017, 7, 20716-20723. [CrossRef]

48. Zakharova, K.; Sterflinger, K.; Razzazi-Fazeli, E.; Noebauer, K.; Marzban, G. Global proteomics of the extremophile black fungus Cryomyces antarcticus using 2D-electrophoresis. Nat. Sci. 2014, 6, 978-995. [CrossRef]

49. Pacelli, C.; Selbmann, L.; Moeller, R.; Zucconi, L.; Fujimori, A.; Onofri, S. Cryptoendolithic Antarctic black fungus Cryomyces antarcticus irradiated with accelerated helium ions: Survival and metabolic activity, DNA and ultrastructural damage. Front. Microbiol. 2017, 8, 2002. [CrossRef] [PubMed]

50. Onofri, S.; Selbmann, L.; Pacelli, C.; Zucconi, L.; Rabbow, E.; de Vera, J.P. Survival, DNA, and ultrastructural integrity of a cryptoendolithic Antarctic fungus in Mars and Lunar rock analogs exposed outside the International Space Station. Astrobiology 2019, 19, 170-182. [CrossRef] [PubMed]

51. Vaca, I.; Chávez, R. Bioactive compounds produced by Antarctic filamentous fungi. In Fungi of Antarctica. Diversity, Ecology and Biotechnological Application; Rosa, L.H., Ed.; Springer Nature: Basel, Switzerland, 2019; pp. 265-283. ISBN 978-3-030-18366-0.

52. Silver, L.L. Challenges of antibacterial discovery. Clin. Microbiol. Rev. 2011, 24, 71-109. [CrossRef]

53. World Health Organization. World Health Organization Antimicrobial Resistance: Global Report on Surveillance. 2014. Available online: https://apps.who.int/iris/bitstream/handle/10665/112642/?sequence=1 (accessed on 3 September 2020).

54. Brunati, M.; Rojas, J.L.; Sponga, F.; Ciciliato, I.; Losi, D.; Göttlich, E.; Hoog, S.; Genilloud, O.; Marinelli, F. Diversity and pharmaceutical screening of fungi from benthic mats of Antarctic lakes. Mar. Genom. 2009, 2, 43-50. [CrossRef]

55. Wu, G.W.; Ma, H.Y.; Zhu, T.J.; Li, J.; Gu, Q.Q.; Li, D.H. Penilactones A and B, two novel polyketides from Antarctic deep-sea derived fungus Penicillium crustosum PRB-2. Tetrahedron 2012, 689, 9745-9749. [CrossRef]

56. Bratchkova, A.; Ivanova, V. Bioactive Metabolites Produced by Microorganisms Collected in Antarctica and the Arctic. Biotechnol. Biotechnol. Equip. 2011, 25, 1-7. [CrossRef]

57. Svahn, K.S.; Chryssanthou, E.; Olsen, B.; Bohlin, L.; Göransson, U. Penicillium nalgiovense Laxa isolated from Antarctica is a new source of the antifungal metabolite amphotericin B. Fungal Biol. Biotechnol. 2015, 2, 1. [CrossRef]

58. Abneuf, M.A.; Krishnan, A.; Aravena, M.G.; Pang, K.L.; Convey, P.; Mohamad-Fauzi, N.; Rizman-Idid, M.; Alias, S.A. Antimicrobial activity of microfungi from maritime Antarctic soil. Czech. Polar Rep. 2016, 6, 141-154. [CrossRef]

59. Wang, J.; Wei, X.; Qin, X.; Tian, X.; Liao, L.; Li, K.; Zhou, X.; Yang, X.; Wang, F.; Zhang, T.; et al. Antiviral merosesquiterpenoids produced by the Antarctic fungus Aspergillus ochraceopetaliformis SCSIO 05702. J. Nat. Prod. 2016, 79, 59-65. [CrossRef]

60. Houbraken, J.; Frisvad, J.C.; Seifert, K.A.; Overy, D.P.; Tuthill, D.M.; Valdez, J.G.; Samson, R.A. New penicillin-producing Penicillium species and an overview of section Chrysogena. Persoonia 2012, 29, 78-100. [CrossRef] [PubMed]

61. Santiago, I.F.; Alves, T.M.; Rabello, A.; Junior, P.A.S.; Romanha, A.J.; Zani, C.L.; Rosa, C.A.; Rosa, L.H. Leishmanicidal and antitumoral activities of endophytic fungi associated with the Antarctic angiosperms 
Deschampsia antarctica Desv. and Colobanthus quitensis (Kunth) Bartl. Extremophiles 2012, 16, 95-103. [CrossRef] [PubMed]

62. Gonçalves, V.N.; Carvalho, C.R.; Johann, S.; Mendes, G.; Alves, T.M.; Zani, C.L.; Junior, P.A.; Murta, S.M.; Romanha, A.J.; Cantrell, C.L.; et al. Antibacterial, antifungal and antiprotozoal activities of fungal communities present in different substrates from Antarctica. Polar Biol. 2015, 38, 1143-1152. [CrossRef]

63. Li, L.; Li, D.; Luan, Y.; Gu, Q.; Zhu, T. Cytotoxic metabolites from the Antarctic psychrophilic fungus Oidiodendron truncatum. J. Nat. Prod. 2012, 75, 920-927. [CrossRef]

64. Ding, Z.; Li, L.; Che, Q.; Li, D.; Gu, Q.; Zhu, T. Richness and bioactivity of culturable soil fungi from the Fildes Peninsula, Antarctica. Extremophiles 2016, 20, 425-435. [CrossRef]

65. Alborés, S.; Sanguiñedo, P.; Held, B.H.; Cerdeiras, M.P.; Blanchette, R.A. Biodiversity and antimicrobial activity of Antarctic fungi from the Fildes Peninsula, King George Island. Sydowia 2018, 70, 185-192.

66. Li, W.T.; Luo, D.; Huang, J.N.; Wang, L.L.; Zhang, F.G.; Xi, T.; Liao, J.M.; Lu, Y.Y. Antibacterial constituents from Antarctic fungus, Aspergillus sydowii SP-1. Nat. Prod. Res. 2018, 32, 662-667. [CrossRef]

67. Li, Y.; Sun, B.; Liu, S.; Jiang, L.; Liu, X.; Zhang, H.; Che, Y. Bioactive asterric acid derivatives from the Antarctic ascomycete fungus Geomycessp. J. Nat. Prod. 2008, 71, 1643-1646. [CrossRef]

68. Wang, J.; He, W.; Qin, X.; Wei, X.; Tian, X.; Liao, L.; Liao, S.; Yang, B.; Tu, Z.; Chen, B.; et al. Three new indolyl diketopiperazine metabolites from the Antarctic soil-derived fungus Penicillium sp. SCSIO 05705. RSC Adv. 2015, 5, 68736-68742. [CrossRef]

69. Maggi, O.; Tosi, S.; Angelova, M.; Lagostina, E.; Fabbri, A.A.; Pecoraro, L.; Altobelli, E.; Picco, A.M.; Savino, E.; Branda, E.; et al. Adaptation of fungi, including yeasts, to cold environments. Plant Biosyst. 2013, 147, 247-258. [CrossRef]

70. Rusinova-Videva, S.; Kambourova, M.; Alipieva, K.; Nachkova, S.; Simova, S. Metabolic profiling of Antarctic yeasts by proton nuclear magnetic resonance-based spectroscopy. Biotechnol. Biotechnol. Equip. 2019, 33, 12-19. [CrossRef]

71. Vishniac, H.S.; Hempfling, W.P. Cryptococcus vishniacii sp. nov., an Antarctic yeast. Int. J. Syst. Evol. Microbiol. 1979, 29, 153-158. [CrossRef]

72. Vishniac, H.S. Cryptococcus friedmannii, a new species of yeast from the Antarctic. Mycologia 1985, 77, 149-153. [CrossRef] [PubMed]

73. Vishniac, H.S.; Kurtzman, C.P. Cryptococcus antarcticus sp. nov. and Cryptococcus albidosimilis sp. nov., basidioblastomycetes from Antarctic soils. Int. J. Syst. Evol. Microbiol. 1992, 42, 547-553. [CrossRef]

74. Rusinova-Videva, S.; Pavlova, K.; Georgieva, K. Effect of different carbon sources on biosynthesis of exopolysaccharide from Antarctic strain Cryptococcus laurentii AL62. Biotechnol. Biotechnol. Equip. 2011, 25, 80-84. [CrossRef]

75. Ocampo-Friedmann, R.; Friedmann, E.I. Biologically active substances produced by Antarctic cryptoendolithic fungi. Antarct. J. U. S. 1993, 28, 252-254.

76. Purić, J.; Vieira, G.; Cavalca, L.B.; Sette, L.D.; Ferreira, H.; Vieira, M.L.C.; Sass, D.C. Activity of Antarctic fungi extracts against phytopathogenic bacteria. Lett. Appl. Microbiol. 2018, 66, 530-536. [CrossRef]

77. Ferrarezi, J.H.; dos Santos, J.A.; Sette, L.D.; Ferreira, H.; Sass, D.C. Anti-Xanthomonas activity of Antarctic fungi crude extracts. Afr. J. Biotechnol. 2019, 18, 713-718.

78. Gomes, E.C.; Godinho, V.M.; Silva, D.A.; de Paula, M.T.; Vitoreli, G.A.; Zani, C.L.; Alves, T.M.A.; Junior, P.A.S.; Murta, S.M.F.; Barbosa, E.C.; et al. Cultivable fungi present in Antarctic soils: Taxonomy, phylogeny, diversity, and bioprospecting of antiparasitic and herbicidal metabolites. Extremophiles 2018, 22, 381-393. [CrossRef]

79. Melo, I.S.; Santos, S.N.; Rosa, L.H.; Parma, M.M.; Silva, L.J.; Queiroz, S.C.; Pellizari, V.H. Isolation and biological activities of an endophytic Mortierella alpina strain from the Antarctic moss Schistidium antarctici. Extremophiles 2014, 18, 15-23. [CrossRef]

80. Ferreira, E.M.S.; Resende, D.A.; Vero, S.; Pimenta, R.S. The Use of Psychrophilic Antarctic Yeast in the Biological Control of Post-harvest Diseases of Fruits Stored at Low Temperatures. In Fungi of Antarctica. Diversity, Ecology and Biotechnological Application; Rosa, L.H., Ed.; Springer Nature: Basel, Switzerland, 2019; pp. 243-263. ISBN 978-3-030-18366-0.

81. Vero, S.; Garmendia, G.; González, M.B.; Bentancur, O.; Wisniewski, M. Evaluation of yeasts obtained from Antarctic soil samples as biocontrol agents for the management of postharvest diseases of apple (Malus $\times$ domestica). FEMS Yeast Res. 2013, 13, 189-199. [CrossRef] [PubMed] 
82. Arrarte, E.; Garmendia, G.; Rossini, C.; Wisniewski, M.; Vero, S. Volatile organic compounds produced by Antarctic strains of Candida sake play a role in the control of postharvest pathogens of apples. Biol. Control 2017, 109, 14-20. [CrossRef]

83. Pacelli, C.; Cassaro, A.; Maturilli, A.; Timperio, A.M.; Gevi, F.; Cavalazzi, B.; Stefan, M.; Ghica, D.; Onofri, S. Multidisciplinary characterization of melanin pigments from the black fungus Cryomyces antarcticus. Appl. Microbiol. Biotechnol. 2020, 104, 6385-6395. [CrossRef] [PubMed]

84. Pombeiro-Sponchiado, S.R.; Sousa, G.S.; Andrade, J.C.R.; Lisboa, H.F.; Gonçalves, R.C. Production of melanin pigment by fungi and its biotechnological applications. In Melanin; Blumenberg, M., Ed.; IntechOpen: London, UK, 2017; Chapter 4. [CrossRef]

85. Kuncheva, M.; Panchev, I.; Pavlova, K.; Russinova-Videva, S.; Georgieva, K.; Dimitrova, S. Functional Characteristics of an Exopolysaccharide from Antarctic Yeast Strain Cryptococcus Laurentii AL 62. Biotechnol. Biotechnol. Equip. 2013, 27, 4098-4102. [CrossRef]

86. Hawksworth, D.L. The fungal dimension of biodiversity: Magnitude, significance, and conservation. Mycol. Res. 1991, 95, 641-655. [CrossRef]

87. Blackwell, M. The Fungi: 1, 2, 3 ... 5.1 million species? Am. J. Bot. 2011, 98, 426-438. [CrossRef]

88. Wu, B.; Hussain, M.; Zhang, W.; Stadler, M.; Liu, X.; Xiang, M. Current insights into fungal species diversity and perspective on naming the environmental DNA sequences of fungi. Mycology 2019, 10, 127-140. [CrossRef]

89. De Sousa, J.R.; Gonçalves, V.N.; de Holanda, R.A.; Santos, D.A.; Bueloni, C.F.; Costa, A.O.; Petry, M.V.; Rosa, C.A.; Rosa, L.H. Pathogenic potential of environmental resident fungi from ornithogenic soils of Antarctica. Fungal Biol. 2017, 121, 991-1000. [CrossRef]

90. Lohan, D.; Johnston, S. Bioprospecting in Antarctica; United Nations University-Institute of Advanced Studies: Yokohama, Japan, 2005; p. 31. Available online: https://www.cbd.int/financial/bensharing/g-absantarctic.pdf (accessed on 3 September 2020).

(C) 2020 by the authors. Licensee MDPI, Basel, Switzerland. This article is an open access article distributed under the terms and conditions of the Creative Commons Attribution (CC BY) license (http://creativecommons.org/licenses/by/4.0/). 